Supporting Information

\title{
A Quantum Chemical Study of the Catalysis for Cytidine Deaminase:
}

\section{Contribution of the Extra Water Molecule}

\author{
Toshiaki Matsubara, ${ }^{*},+\infty$ Masashi Ishikura, ${ }^{*}$ and Misako Aida ${ }^{*},+$, \\ ${ }^{\dagger}$ Center for Quantum Life Sciences and Graduate School of Science, Hiroshima University, 1-3-1, \\ Kagamiyama, Higashi-Hiroshima 739-8530, Japan.
}

\section{Contents}

1. Figure $\mathrm{S} 1$

2. Figure $\mathrm{S} 2$

3. Cartesian Coordinates of Optimized Structures 


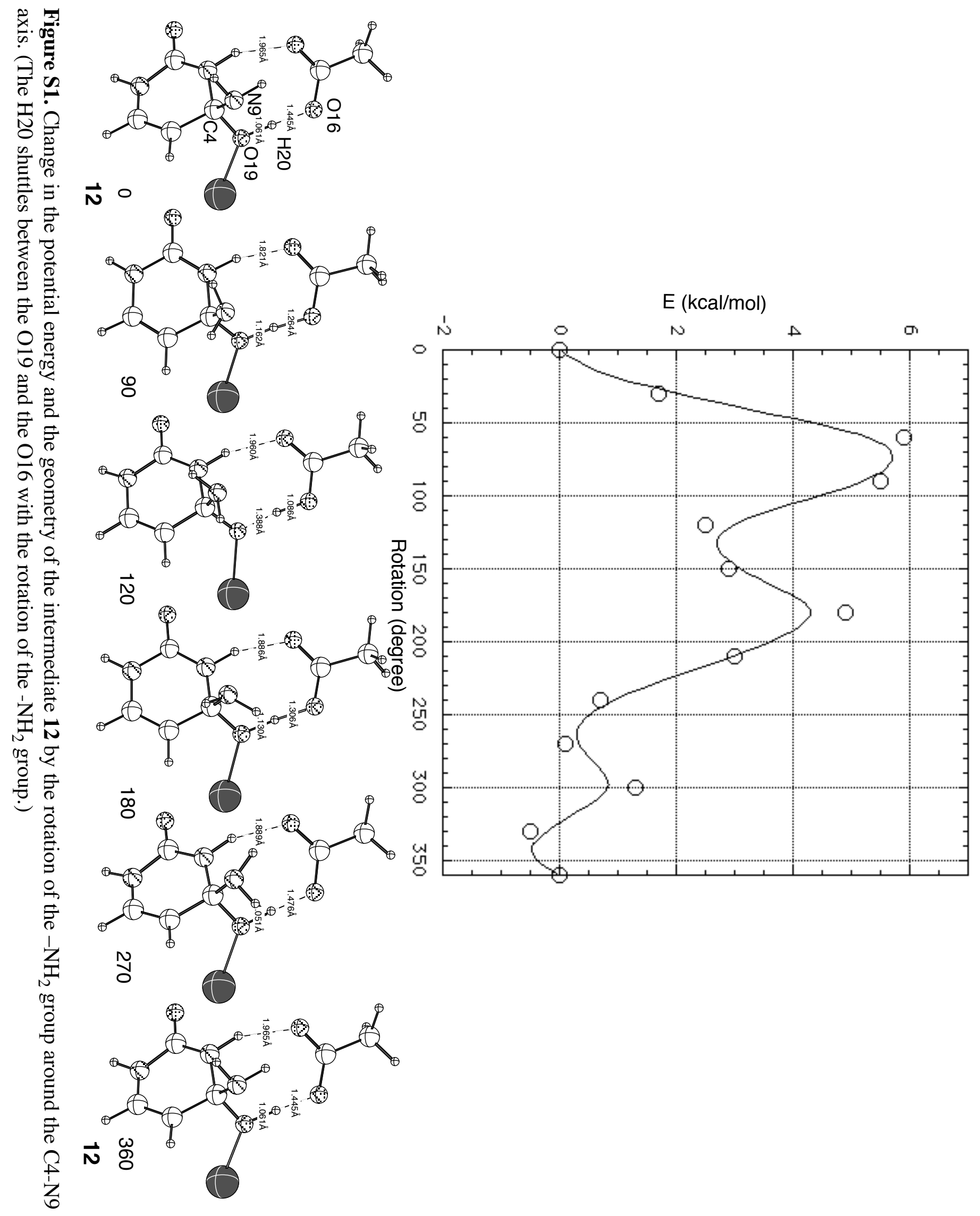




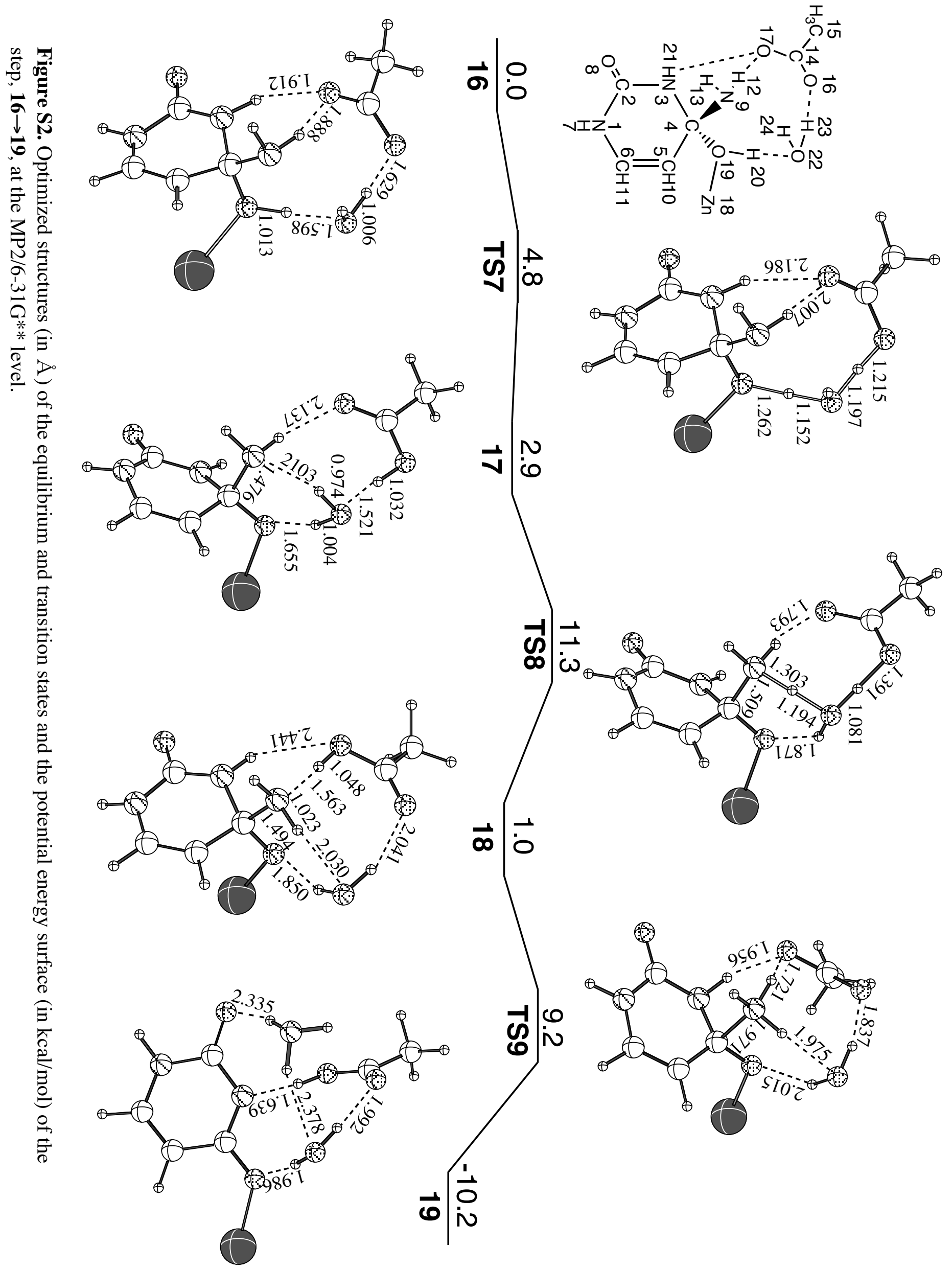




\section{Cartesian Coordinates (in $\AA$ ) of Optimized Structures}

\begin{abstract}
B3LYP level
Structure 1

C $1.131180-0.253174-0.003715$

N $\quad 0.081867-1.053065 \quad 0.002632$

C -1.187789 -0.530206 -0.000093

$\begin{array}{lll}\mathrm{N}-1.279639 & 0.896786 & 0.002452\end{array}$

C - $0.199486 \quad 1.715492 \quad 0.001676$

C $1.052592 \quad 1.186232-0.002099$ O -2.224007 -1.174119 0.001105

H $1.9340691 .814215-0.013349$ $\mathrm{H}-0.391726 \quad 2.783621 \quad 0.003026$ $\mathrm{H}-2.221997 \quad 1.261981 \quad 0.005191$ N $2.358696-0.845409-0.040762$

H $2.378427-1.845616 \quad 0.091165$

H $\quad 3.187839-0.319499 \quad 0.180260$
\end{abstract}

\section{Structure 2a}

N $2.0131120 .350632-0.010087$

C $2.025522-1.006028-0.016755$

C $0.857635-1.699329-0.003039$

H $2.998237-1.486863-0.032088$

$\begin{array}{lll}\text { C }-0.355237 & -0.918936 & 0.014938\end{array}$

H $\quad 0.841172-2.781485-0.003273$

H $2.867747 \quad 0.889672-0.020552$

$\mathrm{N}-0.357527 \quad 0.412965 \quad 0.015942$

$\mathrm{N}-1.553994-1.538220 \quad 0.045427$

$\begin{array}{llll}\text { C } & 0.815677 & 1.123796 & 0.007981\end{array}$

H $-2.1801921 .021692-0.153062$

$\begin{array}{llll}O & 0.905864 & 2.340961 & 0.014846\end{array}$

$\mathrm{H}-1.617830-2.537007-0.054088$

H - $2.390452-0.948370-0.007240$

O $-3.139518 \quad 0.801702-0.121163$

H-3.422171 $1.126394 \quad 0.743129$

\section{Structure 2aw}

$\mathrm{N}-2.166426 \quad 0.156023 \quad 0.179255$

C - $1.934874-1.208593 \quad 0.153794$

C - $0.702637-1.704897-0.015739$

$\mathrm{H}-2.812234-1.8362820 .266618$

C $0.467633-0.770676-0.119044$

H -0.528891 -2.772628 -0.070955

$\mathrm{H}-3.0883820 .533850 \quad 0.331118$

$\mathrm{N} \quad 0.026556 \quad 0.554260-0.471619$

N $1.424500-1.225442-1.095464$

C - $-1.190631 \quad 1.099547-0.136253$

O $-1.432088 \quad 2.297432-0.156777$

H $1.769527-2.150226-0.854519$

H $\quad 2.217483-0.586185-1.125450$

O $1.087058-0.603594 \quad 1.223157$

H $1.347584-1.4810261 .538839$

H $\quad 0.777146 \quad 1.231841-0.573377$

H $2.419323 \quad 0.6864790 .814366$

$\begin{array}{llll}\mathrm{O} & 2.856614 & 1.229258 & 0.130078\end{array}$

H $2.976418 \quad 2.103245 \quad 0.519948$

Structure 2b
$\begin{array}{lll}C-0.536957 & 0.939202 & 0.000021\end{array}$ C $-0.358063-0.494825-0.000062$

N $0.822511-1.094121-0.000107$

C $1.974045-0.351617-0.000086$

O $3.112436-0.796706-0.000029$

$\begin{array}{llll}\mathrm{N} & 1.810831 & 1.069078 & 0.000004\end{array}$

H $2.672808 \quad 1.5958620 .000069$

C $\quad 0.599235 \quad 1.682075 \quad 0.000060$

H $\quad 0.598735 \quad 2.767690 \quad 0.000132$

$\mathrm{N}-1.460375-1.280855-0.000093$

$\mathrm{H}-1.522499 \quad 1.385892 \quad 0.000054$

H -2.394302 -0.886686 -0.000126

$\mathrm{H}-1.313613-2.277862-0.000205$

$\begin{array}{llll}\mathrm{O}-4.005454 & 0.238069 & 0.000169\end{array}$

$\mathrm{H}-4.588913 \quad 0.188256-0.766924$

$\mathrm{H}-4.588395 \quad 0.188220 \quad 0.767654$

H - $0.046183-2.000265-0.000349$

\section{Structure 2bw}

$\begin{array}{llll}\mathrm{N} & 2.246860 & 0.973331 & 0.343492\end{array}$

C $1.091596 \quad 1.683609 \quad 0.291301$

C $-0.064843 \quad 1.072026-0.078906$

$\begin{array}{llll}\mathrm{H} & 1.150471 & 2.735115 & 0.553637\end{array}$

C $0.044193-0.325577-0.409081$

$\mathrm{H}-1.009011 \quad 1.596497-0.149156$

H $3.1213591 .403742 \quad 0.611216$

O -3.328236 $0.851200-0.744549$

N $1.163311-1.018170-0.372806$

$\mathrm{N}-1.108632-1.004763-0.746482$

C $2.337786-0.411628 \quad 0.011837$

O $3.426538-0.956725 \quad 0.086254$

$\begin{array}{lll}\mathrm{H}-3.479434 & 0.363580 & 0.094946\end{array}$

$\mathrm{H}-1.881337-0.441785-1.108725$

$\mathrm{H}-0.934845-1.895406-1.195585$

H -2.350946 -1.183496 0.895128

O $-3.128929-0.858297 \quad 1.390452$

$\mathrm{H}-2.763552-0.467406 \quad 2.194115$

H $-4.170850 \quad 0.836366-1.213171$

\section{Structure 3aw}

$\begin{array}{llll}\mathrm{N} & 2.388366 & 0.519437 & 0.061010\end{array}$

C $2.583217-0.820601-0.050333$

C $\quad 1.520811-1.662957-0.107007$

H $3.612094-1.163284-0.086004$

C $\quad 0.206823-1.064338-0.048137$

H $1.653982-2.733921-0.190079$

H $3.162302 \quad 1.167636 \quad 0.102064$

$\begin{array}{llll}\mathrm{N} & 0.032047 & 0.254958 & 0.044337\end{array}$

$\mathrm{N}-0.883386-1.847934-0.081041$

$\begin{array}{llll}\text { C } & 1.099097 & 1.112998 & 0.108799\end{array}$

$\begin{array}{llll}\text { O } & 1.009672 & 2.329332 & 0.202927\end{array}$

H $-0.776958-2.845294-0.166321$

$\mathrm{H}-1.833667-1.449198 \quad 0.013191$

$\begin{array}{lll}\mathrm{O}-3.448309 & -0.712369 & 0.254835\end{array}$

H -3.583790 $-0.631588 \quad 1.207199$

$\mathrm{H}-1.465706 \quad 1.228373-0.345736$

H -3.220843 $0.208867-0.036131$
O -2.362152 $1.625218-0.479840$

H $-2.359989 \quad 2.405118 \quad 0.088364$

Structure $\mathbf{3 b}$

C $-1.481664 \quad 1.317499-0.000198$

C -1.796456 -0.073028 -0.000095

$\mathrm{N}-0.923798-1.049645 \quad 0.000036$

C $0.422963-0.757337 \quad 0.000083$

$\begin{array}{llll}O & 1.311074-1.606566 & 0.000207\end{array}$

N $\quad 0.772357 \quad 0.614269-0.000012$

H $\quad 1.794954 \quad 0.802844 \quad 0.000025$

C $-0.147043 \quad 1.604272-0.000148$

H $\quad 0.237756 \quad 2.619336-0.000214$

$\begin{array}{llll}\mathrm{N} & 3.592456 & 0.317916 & 0.000185\end{array}$

H -2.250355 $2.076805-0.000307$

H $4.1882610 .412617-0.818078$

H $\quad 4.188009 \quad 0.412695 \quad 0.818623$

O -3.100569 -0.399298 -0.000143

$\mathrm{H}-3.127552-1.371343-0.000061$

H $3.210985-0.6322590 .000181$

Structure $\mathbf{3 b w}$

N $2.533338-0.220817 \quad 0.296399$

C $2.114436-1.486920 \quad 0.039690$

C $\quad 0.827700-1.711518-0.335935$

H $\quad 2.850214-2.276845 \quad 0.149359$

C - $0.012954-0.549840-0.435004$

H $\quad 0.446572-2.698448-0.556870$

H $\quad 3.483876-0.025350 \quad 0.578994$

O $-1.274079-0.756963-0.803390$

N $0.389255 \quad 0.678491-0.183279$

$\mathrm{N}-2.525007 \quad 1.513609-0.596509$

C $1.689740 \quad 0.922471 \quad 0.198272$

$\begin{array}{llll}O & 2.157182 & 2.020436 & 0.453400\end{array}$

$\mathrm{H}-2.699608-0.987881 \quad 0.728042$

$\mathrm{H}-1.7409332 .070422-0.260808$

H -3.062221 $2.058335-1.263833$

H -3.115888 $1.255955 \quad 0.195067$

O -3.467741 $-0.670022 \quad 1.229985$

$\mathrm{H}-4.212203-1.1490690 .845538$

$\mathrm{H}-1.7693290 .161136-0.873869$

\section{Structure $4 a$}

$\begin{array}{llll}\text { N } & 1.599629 & 0.881933 & 0.023953\end{array}$

C $\quad 0.534474 \quad 1.763778-0.074485$

C $-0.735088 \quad 1.339752-0.105877$

H $0.803219 \quad 2.813409-0.126411$

C - $-1.074295-0.121913 \quad 0.019632$

$\mathrm{H}-1.5583412 .041233-0.155867$

H $2.552881 \quad 1.205148 \quad 0.074024$

O -1.651679 $-0.298716 \quad 1.303100$

N $\quad 0.139475-0.905542-0.173740$

$\mathrm{N}-2.043089-0.650050-0.941643$

C $1.445877-0.497990-0.021732$

H $\quad 0.025501-1.910510-0.186001$

O $2.397363-1.2648100 .020485$

H -2.080168 -1.169140 1.287777 
$\mathrm{H}-2.912457-0.128261-0.858405$

$\mathrm{H}-1.694019-0.529819-1.889017$

\section{Structure $\mathbf{4 b}$}

C -1.114264 $-0.315629 \quad 0.000092$ $\mathrm{N}-0.052707-1.069943-0.000017$

C $1.201346-0.497634-0.000029$

N $1.233041 \quad 0.932297-0.000024$

C $\quad 0.123398 \quad 1.7059890 .000078$

C - $1.107030 \quad 1.121793 \quad 0.000150$

O $2.258008-1.101173-0.000299$

$\mathrm{H}-2.013233 \quad 1.714808 \quad 0.000212$

$\begin{array}{llll}H & 0.270615 & 2.780931 & 0.000080\end{array}$

H $2.159858 \quad 1.336670-0.000110$

O -2.292792 $-0.975633 \quad 0.000078$

H -3.022003 -0.341556 0.000132

\section{Structure 5aw}

$\mathrm{N}-2.023948 \quad 1.024512 \quad 0.003290$ C - $-0.861298 \quad 1.778944 \quad 0.046282$

$\begin{array}{llll}\text { C } & 0.351411 & 1.214805 & 0.093227\end{array}$ $\mathrm{H}-1.005124 \quad 2.853997 \quad 0.036962$

C $0.526213-0.281033 \quad 0.054833$

H $\quad 1.253008 \quad 1.813756 \quad 0.106445$

H -2.929760 $1.453585-0.102396$

O $1.144290-0.743156-1.124366$

$\mathrm{N}-0.775649-0.930821 \quad 0.126122$

N $1.403922-0.687599 \quad 1.179497$

C -2.022229 $-0.363039-0.037853$

H - $0.773135-1.920764-0.085753$

O $-3.048215-1.015072-0.166127$

H $2.055355-0.376694-1.120466$

H $0.973075-0.417602 \quad 2.061439$

H $1.481606-1.7046191 .174795$

$\begin{array}{llll}\text { H } & 3.058041 & 0.017811 & 0.522410\end{array}$

O $3.572724 \quad 0.331755-0.257071$

H $4.341691-0.248392-0.314224$

\section{Structure $\mathbf{5 b}$}

C -1.131223 -0.262538 -0.000009 $\mathrm{N}-0.087814-1.049898-0.000181$

C $1.183115-0.510050-0.000344$

$\begin{array}{llll}\mathrm{N} & 1.242211 & 0.915034 & 0.000061\end{array}$

C $\quad 0.147984 \quad 1.717165 \quad 0.000248$ $\begin{array}{llll}\mathrm{C}-1.097301 & 1.168076 & 0.000162\end{array}$

O $2.222489-1.143440-0.000017$ $\mathrm{H}-2.002269 \quad 1.758152 \quad 0.000298$

$\begin{array}{llll}H & 0.325964 & 2.787318 & 0.000474\end{array}$

H $\quad 2.177444 \quad 1.299386 \quad 0.000186$

O -2.347958 -0.829869 -0.000021

H -2.193616 -1.790246 -0.000148

\section{Structure 5bw}

C - $0.418742-0.860811-0.002631$

$\mathrm{N}-0.340661 \quad 0.458838-0.009171$

C $0.882871 \quad 1.095485-0.003183$

$\begin{array}{llll}\mathrm{N} & 2.017658 & 0.240119 & 0.003272\end{array}$

C $1.937967-1.115887 \quad 0.009731$

C $0.724110-1.727577 \quad 0.009464$

O $1.045951 \quad 2.303192-0.000941$

H $\quad 0.607393-2.801718 \quad 0.013348$

H $2.876957-1.6591990 .014815$

H $2.909042 \quad 0.716933 \quad 0.003904$

O -1.608142 -1.442084-0.006092

H -2.314391 -0.729389-0.026292

O -3.066266 $0.772276-0.081731$

H -2.144924 1.121169-0.116479

\section{H -3.402634 $1.045178 \quad 0.781820$}

\section{Structure 6a}

N $1.654191 \quad 1.157946-0.000557$

C $\quad 0.364029 \quad 1.640643 \quad 0.000194$

$\begin{array}{llll}\text { C }-0.709363 & 0.821214 & 0.000573\end{array}$

$\mathrm{H} \quad 0.274608 \quad 2.721503 \quad 0.000161$

C -0.504949 $-0.622036 \quad 0.000352$

$\mathrm{H}-1.731685 \quad 1.185123 \quad 0.000640$

H $2.449355 \quad 1.780057 \quad 0.000344$

$\begin{array}{lll}\mathrm{O}-1.382602-1.479090 & 0.000035\end{array}$

N $\quad 0.848197-1.010737 \quad 0.000172$

$\mathrm{N}-3.956048 \quad 0.317351-0.000594$

C $1.971455-0.198023-0.000147$

H $\quad 1.030192-2.007242 \quad 0.000305$

O $3.116324-0.611720-0.000175$

H -3.326353 -0.486640 -0.005791

$\mathrm{H}-4.553262 \quad 0.214019 \quad 0.816621$

H -4.564045 $0.216962-0.810131$

\section{Structure 6aw}

$\mathrm{N}-1.994561 \quad 1.077641-0.124152$

C $-0.8058901 .607654-0.570742$

C $\quad 0.284929 \quad 0.839564-0.775453$

$\mathrm{H}-0.808757 \quad 2.678505-0.740325$

C $0.210994-0.587868-0.509776$

H $1.229769 \quad 1.251073-1.102008$

$\mathrm{H}-2.801284 \quad 1.661291 \quad 0.042673$

O $1.122270-1.403374-0.659611$

$\mathrm{N}-1.041151-1.030243-0.070667$

N $2.086375 \quad 0.367220 \quad 2.089370$

C -2.177785 -0.270996 0.167576

$\mathrm{H}-1.122076-2.016980 \quad 0.142950$

O -3.229130 $-0.726250 \quad 0.577266$

H $2.724288-0.551151-0.801430$

H $\quad 2.379076 \quad 1.184972 \quad 2.619964$

H $2.353543-0.437463 \quad 2.652304$

H $\quad 2.672780 \quad 0.340647 \quad 1.252667$

O $3.4423290 .111377-0.722776$

H $\quad 4.260778-0.397377-0.757322$

\section{Structure 6bw}

C $-0.444221-1.004836-0.006320$

$\mathrm{N}-0.319149 \quad 0.388876-0.014285$

C $0.851075 \quad 1.126729-0.005550$

$\begin{array}{llll}\mathrm{N} & 2.002408 & 0.331331 & 0.007908\end{array}$

C $1.979774-1.040215 \quad 0.014815$

C $0.817276-1.730234 \quad 0.009947$

$\begin{array}{llll}\text { O } & 0.903012 & 2.341879 & -0.006201\end{array}$

H $\quad 0.790219-2.8106120 .015053$

H $2.950096-1.524853 \quad 0.024599$

$\begin{array}{llll}\text { H } & 2.871717 & 0.845045 & 0.012414\end{array}$

O - $1.557703-1.535580-0.011984$

H -2.887969 $-0.176126-0.102663$

O -3.100965 $0.779428-0.075354$

$\mathrm{H}-1.208076 \quad 0.910005-0.036367$

$\mathrm{H}-3.476976 \quad 0.920615 \quad 0.802558$

\section{Structure 7}

C -1.294591 $-0.347804 \quad 0.000188$

$\mathrm{N}-0.033041-0.987360-0.000131$

C $1.223421-0.407544-0.000046$

$\begin{array}{llll}\mathrm{N} & 1.175033 & 0.987782 & 0.000118\end{array}$

C $0.004852 \quad 1.7099520 .000252$

$\begin{array}{lll}C-1.204768 & 1.109272 & 0.000206\end{array}$

O $2.270095-1.028457-0.000281$

$\mathrm{H}-2.124406 \quad 1.677223 \quad 0.000214$
$\begin{array}{llll}H & 0.124033 & 2.787964 & 0.000331\end{array}$ $\begin{array}{llll}H & 2.076057 & 1.443110 & 0.000149\end{array}$ O -2.319711 -1.008322 -0.000201

Structure 8

N -2.764591 -0.535544 -0.840252

C -2.304240 $0.660470-1.364319$

C - $1.286272 \quad 1.334419-0.813747$

$\mathrm{H}-2.823147 \quad 1.011155-2.249633$

C - $0.545476 \quad 0.793785 \quad 0.375565$

$\mathrm{H}-0.9204732 .257562-1.244115$

H -3.498384 -1.063208 -1.286746

$\begin{array}{llll}O & 0.785529 & 0.378918 & 0.046994\end{array}$

$\mathrm{N}-1.236204-0.3681890 .902287$

N $-0.393697 \quad 1.859080 \quad 1.387048$

C -2.216124-1.126734 0.289313

H $-0.758032-0.876815 \quad 1.635376$

O -2.601133 -2.201126 0.722179

H $1.272688 \quad 1.196245-0.221900$

$\mathrm{H}-1.314256 \quad 2.185251 \quad 1.674157$

H $\quad 0.066076 \quad 1.469839 \quad 2.209819$

$\begin{array}{llll}\mathrm{H} & 0.922222 & 2.974441 & 0.478507\end{array}$

O $1.588306 \quad 2.984334-0.245004$

H $\quad 2.425387 \quad 3.240896 \quad 0.161240$

Zn $2.510680-1.278381-0.213817$

\section{Structure 9}

N 2.716851-1.360941 0.440140

C $1.360102-1.578243 \quad 0.439420$

C $0.494403-0.685717-0.086906$

H $\quad 1.033191-2.5082450 .891216$

C $1.006926 \quad 0.538854-0.666914$

H $-0.577109-0.851006-0.068128$

H $3.356498-2.0272210 .848960$

O $0.335404 \quad 1.431984-1.198444$

N $\quad 2.394474 \quad 0.669887-0.624138$

$\mathrm{N}-0.398486 \quad 1.844660 \quad 2.065591$

C $3.315520-0.215501-0.078087$

H $2.782309 \quad 1.524366-1.006050$

O $4.515677-0.022600-0.058506$

$\mathrm{H}-1.328227 \quad 1.349528-0.894274$

$\mathrm{H}-0.819145 \quad 1.516670 \quad 2.932092$

$\mathrm{H}-0.185814 \quad 2.828854 \quad 2.212207$

$\mathrm{H}-1.134107 \quad 1.810380 \quad 1.361179$

O $-2.264904 \quad 1.282094-0.567421$

$\mathrm{H}-2.744306 \quad 2.034610-0.940411$

Zn -3.037476 -0.788045 -0.051935

\section{Structure 10}

$\begin{array}{lll}\text { C - }-0.422127 & 0.155851 & 0.753823\end{array}$

$\begin{array}{lllll}\mathrm{N} & 0.098630 & 1.347438 & 0.080631\end{array}$

C - $0.6045892 .432552-0.344366$

$\mathrm{N}-1.935151 \quad 2.476865 \quad 0.105379$

C -2.470979 $1.539733 \quad 0.964956$

C -1.796392 $0.431833 \quad 1.303082$

H $\quad 1.083574 \quad 1.277050-0.245858$

O $-0.162863 \quad 3.354239-1.025959$

$\mathrm{H}-2.4402553 .304634-0.164947$

$\begin{array}{lll}\mathrm{H}-3.473948 & 1.747098 & 1.325453\end{array}$

$\mathrm{H}-2.208404-0.298694 \quad 1.986616$

N $0.469209-0.299655 \quad 1.813813$

H $\quad 0.4710820 .417502 \quad 2.535560$

H $\quad 1.436637-0.353522 \quad 1.450453$

O -0.551987 -0.916059-0.201269

H $\quad 0.182521-1.587937-0.092382$

O $1.329316-2.736330 \quad 0.377041$

H $\quad 2.117640-2.126404 \quad 0.373584$ 
H $\quad 1.021768-2.645104 \quad 1.291572$

$\begin{array}{llll}\text { O } & 2.807711 & 0.982021 & -0.654163\end{array}$

C $3.489397 \quad 0.035660-0.206192$

C $4.918203-0.139349-0.749254$

O $3.131913-0.8210290 .674309$

H $4.992571-1.095193-1.280708$

H $\quad 5.630003-0.181430 \quad 0.082306$

H $5.1852280 .673661-1.428056$

Zn -2.518034 -1.648154 -0.784144

Structure 11

C - $1.053955 \quad 0.664454 \quad 0.016738$

$\begin{array}{llll}\mathrm{N} & 0.221097 & 0.972895 & -0.314243\end{array}$

C $0.614912 \quad 2.251169-0.588025$

$\mathrm{N}-0.398073 \quad 3.243030-0.576325$

C - $1.702850 \quad 2.969422-0.282928$

C -2.076156 $1.704741 \quad 0.016328$

H $1.260530-0.193044-0.772823$

O $1.767542 \quad 2.604987-0.835536$

$\mathrm{H}-0.0755334 .180771-0.762848$

$\mathrm{H}-2.390544 \quad 3.809675-0.302566$

$\mathrm{H}-3.096773 \quad 1.442034 \quad 0.259103$

$\begin{array}{llll}\mathrm{N} & 2.872062 & 1.325828 & 2.169012\end{array}$

H $3.476171 \quad 0.560263 \quad 1.869737$

$\begin{array}{llll}H & 2.656466 & 1.818067 & 1.300486\end{array}$

O -1.358969 $-0.529663 \quad 0.333109$

$\mathrm{H}-0.100025-0.8872601 .848269$

O $0.618869-1.008838 \quad 2.490614$

H $1.363321-1.266416 \quad 1.919995$

H $2.005103 \quad 0.842243 \quad 2.417900$

O $1.844507-0.839958-1.329590$

C $2.648469-1.613595-0.633953$

C $3.524080-2.470011-1.534978$

O $2.723736-1.677448 \quad 0.588390$

H $\quad 4.169763-3.107367-0.930224$

H $\quad 4.130153-1.828188-2.182053$

H $2.894721-3.084976-2.185827$

Zn $-3.055048-1.683593-0.112108$

\section{Structure 12}

C -0.385375 $0.317610 \quad 0.943513$

$\mathrm{N}-0.022348 \quad 1.465159 \quad 0.081064$

C - $0.834185 \quad 2.185161-0.737142$

$\mathrm{N}-2.200654 \quad 1.954771-0.528433$

C -2.683442 1.1204290 .466551

$\begin{array}{lll}C-1.871749 & 0.339312 & 1.191093\end{array}$

H $\quad 0.989103 \quad 1.559798-0.051794$

O - $0.464313 \quad 3.016572-1.565278$

$\mathrm{H}-2.8137682 .523206-1.088844$

H -3.760759 $1.129402 \quad 0.601830$ H -2.251162 $-0.301534 \quad 1.975678$

$\begin{array}{llll}\mathrm{N} & 0.344199 & 0.390022 & 2.208415\end{array}$

H $\quad 0.132397 \quad 1.297020 \quad 2.622710$

H $1.338337 \quad 0.428147 \quad 1.963445$

O $-0.057206-0.898360 \quad 0.307695$

H $\quad 0.983480-0.985047 \quad 0.117654$

Zn -1.496557 -2.244941 -0.506201

$\begin{array}{llll}\text { O } & 2.795439 & 0.951057 & 0.423554\end{array}$

C $3.125029-0.158840-0.070161$

C $4.566121-0.316942-0.574184$

O $2.375171-1.174493-0.221892$

H $\quad 5.2363740 .365500-0.045470$

H $4.598195-0.065880-1.641338$

H $4.905002-1.350634-0.465820$

Structure 13

C $0.630511-0.0934420 .689628$
N $\quad 0.522303-1.417699 \quad 0.039221$

C $1.503972-2.210244-0.459365$

$\mathrm{N} \quad 2.807128-1.739321-0.237421$

$\begin{array}{lll}\text { C } 3.064969 & -0.458118 & 0.241902\end{array}$

$\begin{array}{llll}\text { C } & 2.081851 & 0.338984 & 0.675528\end{array}$

$\mathrm{H}-0.441418-1.716043-0.131154$

O $1.330280-3.279565-1.046585$

H $3.512206-2.257711-0.736270$

H $\quad 4.110667-0.1635850 .244092$

$\begin{array}{llll}\text { H } & 2.292751 & 1.333033 & 1.050941\end{array}$

$\begin{array}{llll}\mathrm{O}-0.161065 & 0.845616 & 0.067514\end{array}$

$\begin{array}{llll}N & 0.104554 & -0.272593 & 2.065569\end{array}$

H $\quad 0.054961 \quad 0.650778 \quad 2.492392$

C -4.589846 -0.738878 -0.082070

C -3.065906 -0.688043 -0.089389

O $-2.399785-1.701973-0.294239$

$\begin{array}{lll}O-2.606486 & 0.513719 & 0.141635\end{array}$

$\mathrm{H}-4.965273-0.422083 \quad 0.896567$

H -4.989971 -0.039032 -0.822667

H -4.933541-1.750928 -0.300574

H $0.762194-0.8313802 .607736$

$\mathrm{H}-1.523653 \quad 0.583564 \quad 0.128051$

Zn $\quad 0.500878 \quad 2.689891-0.510157$

\section{Structure 14}

$\begin{array}{lll}C-0.556308 & 0.147699 & 0.566320\end{array}$

$\mathrm{N}-0.001914 \quad 1.279386-0.230685$

C - $0.589028 \quad 2.451577-0.585275$

$\begin{array}{lll}\mathrm{N}-1.823019 & 2.695053 & 0.032029\end{array}$

$\begin{array}{lll}\mathrm{C}-2.484837 & 1.736221 & 0.793479\end{array}$

C - $1.919088 \quad 0.561517 \quad 1.094097$

H $0.895399 \quad 1.078130-0.671528$

O $-0.111564 \quad 3.285964-1.356398$

$\mathrm{H}-2.310774 \quad 3.495582-0.335729$

$\mathrm{H}-3.482772 \quad 2.013955 \quad 1.121433$

H -2.456312 -0.181274 1.672282

$\begin{array}{llll}\mathrm{N} & 0.433598 & 0.012546 & 1.726175\end{array}$

H $\quad 0.378884 \quad 0.833752 \quad 2.330713$

O -0.562082 - $1.015796-0.080052$

O $2.834902 \quad 0.477034-0.861748$

C $3.401020-0.251560-0.058108$

C $4.795802-0.801838-0.311676$

O $2.910418-0.6596891 .097475$

H $4.738541-1.887662-0.441550$

H $5.443548-0.610738 \quad 0.549245$

H $5.216075-0.349086-1.210271$

H $\quad 0.123492-0.7941912 .267769$

H $1.926565-0.345128 \quad 1.251026$

Zn -1.906402 -2.364135 -0.553775

\section{Structure 15}

$\begin{array}{lll}\text { C }-0.943338 & 1.007361 & 0.385903\end{array}$

$\begin{array}{llll}\mathrm{N} & 0.250742 & 0.830483 & -0.240637\end{array}$

C $1.0561051 .871856-0.585559$

N $0.598777 \quad 3.171987-0.248954$

C - $0.585424 \quad 3.400261 \quad 0.392108$

$\begin{array}{lll}C-1.382540 & 2.360071 & 0.723280\end{array}$

H $0.768470-0.687988-0.472027$

O $2.141152 \quad 1.784988-1.166723$

H $1.227873 \quad 3.919846-0.498976$

$\mathrm{H}-0.828879 \quad 4.437396 \quad 0.604875$

$\mathrm{H}-2.329067 \quad 2.496035 \quad 1.229025$

$\begin{array}{llll}\mathrm{O}-1.678922 & 0.019008 & 0.674482\end{array}$

Zn -3.200382 -1.086768 -0.180593

O $0.964623-1.676617-0.703973$

C $1.763326-2.2747790 .158521$

C $1.921732-3.755009-0.162934$
O $\quad 2.322549-1.750492 \quad 1.111822$

H $\quad 2.237096-3.882840-1.203186$

H $\quad 0.956701-4.260439-0.054132$

H $2.652627-4.2059560 .509660$

H $\quad 3.889307-0.165381 \quad 0.954484$

$\begin{array}{llll}N & 4.521055 & 0.475502 & 0.469770\end{array}$

H $4.985215-0.117029-0.219084$

H $3.883727 \quad 1.049923-0.086886$

\section{Structure 16}

C -0.492440 $0.197788 \quad 0.793251$

$\begin{array}{llll}\mathrm{N} & 0.055889 & 1.391400 & 0.128641\end{array}$

C - $0.628241 \quad 2.426852-0.426272$

$\mathrm{N}-1.994762 \quad 2.440005-0.113169$

C -2.571216 $1.548388 \quad 0.773756$

$\begin{array}{lll}C-1.902169 & 0.484297 & 1.238658\end{array}$

H $1.064298 \quad 1.351961-0.045062$

O $-0.138725 \quad 3.324927-1.108216$

$\mathrm{H}-2.499116 \quad 3.235342-0.468985$

$\begin{array}{lll}\mathrm{H}-3.602767 & 1.753691 & 1.042486\end{array}$

H - $2.352364-0.2085831 .937286$

N $\quad 0.353529-0.190610 \quad 1.918126$

$\begin{array}{llll}\mathrm{H} & 0.229221 & 0.510611 & 2.645658\end{array}$

H $\quad 1.328926-0.090690 \quad 1.591425$

O $-0.543334-0.889025-0.131945$

$\begin{array}{llll}O & 2.743632 & 0.532940 & 0.488342\end{array}$

C $3.585127-0.168027-0.148201$

C $4.7084760 .604772-0.857610$

O $3.593637-1.423830-0.264287$

H $4.280550 \quad 1.179471-1.687028$

H $5.472189-0.071768-1.248179$

H $\quad 5.159962 \quad 1.326367-0.169034$

$\mathrm{Zn}-2.466430-1.696832-0.747331$

H $2.241776-2.139926 \quad 0.182355$

O $1.355001-2.5595620 .448665$

H $\quad 1.257921-2.339218 \quad 1.386361$

H $\quad 0.230784-1.535876 \quad 0.015480$

\section{Structure 17}

C $0.711887-0.271421 \quad 0.495833$

N $1.162123-1.298070-0.509424$

C $2.346600-1.955282-0.620527$

N $3.243764-1.693370 \quad 0.420635$

C $3.060284-0.626916 \quad 1.304024$

C $\quad 1.915073 \quad 0.062150 \quad 1.360910$

H $\quad 0.549387-1.388041-1.308515$

O $2.642875-2.736396-1.526914$

H $4.169151-2.0524190 .246020$

H $\quad 3.916645-0.399423 \quad 1.931897$

H $1.785363 \quad 0.872048 \quad 2.067285$

$\mathrm{N}-0.326741-0.857400 \quad 1.377954$

H $0.034420-1.715798 \quad 1.790038$ $\mathrm{H}-1.140104-1.111456 \quad 0.809267$

O $0.188599 \quad 0.797566-0.164902$

O -3.107590 -1.438500 0.065538

C $-4.003781-0.634228-0.163077$

C -5.283418 -1.038215 -0.879072

$\begin{array}{lll}\text { O }-3.998929 & 0.643387 & 0.156805\end{array}$

$\mathrm{H}-5.351607-0.507001-1.834011$

H -6.155701 $-0.748605-0.285097$

$\mathrm{H}-5.288290-2.114156-1.056308$

Zn $\quad 1.384844 \quad 2.335846-0.736195$

$\mathrm{H}-3.108265 \quad 0.9361820 .621234$

O $-1.885169 \quad 1.454012 \quad 1.292350$

$\mathrm{H}-1.132676 \quad 1.375972 \quad 0.622539$

$\mathrm{H}-1.575817 \quad 0.742116 \quad 1.885795$ 
Structure 18

C - $0.522316 \quad 0.113826 \quad 0.591791$

$\mathrm{N}-0.580205 \quad 1.348832-0.232213$

C - $1.6193392 .208897-0.427285$

$\mathrm{N}-2.700303 \quad 2.001963 \quad 0.433479$

C - $2.775498 \quad 0.925285 \quad 1.307395$

$\begin{array}{lll}C-1.773805 & 0.046710 & 1.442502\end{array}$

H $\quad 0.207093 \quad 1.466498-0.860237$

O -1.636586 $3.119474-1.252394$

H $-3.507852 \quad 2.567662 \quad 0.228690$

$\mathrm{H}-3.706200 \quad 0.843805 \quad 1.860535$

$\mathrm{H}-1.861367-0.801637 \quad 2.110080$

$\begin{array}{llll}\mathrm{N} & 0.697374 & 0.371175 & 1.523454\end{array}$

H $\quad 0.471383 \quad 1.038444 \quad 2.259478$

H $1.562651 \quad 0.753154 \quad 0.878725$

O $-0.259530-0.988578-0.103954$

O $2.533244 \quad 1.238416-0.016495$

C $3.5215690 .446885-0.232503$

C $4.5242340 .953025-1.275543$

O $3.731336-0.657358 \quad 0.308864$

H $4.0450610 .963866-2.260856$

H $5.405597 \quad 0.309531-1.311478$

H $4.816828 \quad 1.983425-1.049744$

Zn -1.703543 -2.115914 -1.009341

H $2.533184-1.706607 \quad 1.150436$

O $1.754532-2.149952 \quad 1.565449$

H $1.088336-2.112326 \quad 0.850135$

H $1.020461-0.5459531 .901526$

Structure 19

C-1.053955 $0.664454 \quad 0.016738$

$\begin{array}{llll}\mathrm{N} & 0.221097 & 0.972895 & -0.314243\end{array}$

C $0.614912 \quad 2.251169-0.588025$

$\mathrm{N}-0.398073 \quad 3.243030-0.576325$

C - $1.702850 \quad 2.969422-0.282928$

$\begin{array}{llll}\text { C }-2.076156 & 1.704741 & 0.016328\end{array}$

H $1.260530-0.193044-0.772823$

O $1.767542 \quad 2.604987-0.835536$

H - $0.0755334 .180771-0.762848$

$\mathrm{H}-2.390544 \quad 3.809675-0.302566$

$\mathrm{H}-3.096773 \quad 1.442034 \quad 0.259103$

$\begin{array}{llll}\mathrm{N} & 2.872062 & 1.325828 & 2.169012\end{array}$

H $3.476171 \quad 0.560263 \quad 1.869737$

H $2.656466 \quad 1.818067 \quad 1.300486$

O -1.358969 $-0.529663 \quad 0.333109$

H $-0.100025-0.8872601 .848269$

O $\quad 0.618869-1.008838 \quad 2.490614$

H $\quad 1.363321-1.266416 \quad 1.919995$

H $2.005103 \quad 0.842243 \quad 2.417900$

O $1.844507-0.839958-1.329590$

C $2.648469-1.613595-0.633953$

C $3.524080-2.470011-1.534978$

O $2.723736-1.677448 \quad 0.588390$

H $4.169763-3.107367-0.930224$

H $4.130153-1.828188-2.182053$

H $2.894721-3.084976-2.185827$

Zn -3.055048 -1.683593 -0.112108

Structure 20

C - $-0.558825 \quad 0.181308 \quad 0.688707$

$\mathrm{N}-0.212172 \quad 1.515379 \quad 0.170319$

C -1.054586 2.542788 -0.124422

$\mathrm{N}-2.362200 \quad 2.357560 \quad 0.342449$

C -2.735639 $1.282302 \quad 1.128472$

$\begin{array}{lll}\mathrm{C}-1.917837 & 0.241301 & 1.335656\end{array}$

H $\quad 0.764894 \quad 1.624832-0.109667$

O $-0.7390643 .580458-0.701416$
$\begin{array}{lll}\mathrm{H}-2.982450 & 3.132606 & 0.174323\end{array}$ $\begin{array}{lll}\mathrm{H}-3.739230 & 1.327487 & 1.539379\end{array}$ H -2.213767 -0.599380 1.949355

N $0.472299-0.238467 \quad 1.647639$

$\begin{array}{llll}\mathrm{H} & 0.378976 & 0.360548 & 2.466425\end{array}$

H $\begin{array}{lll}1.381446 & 0.023564 & 1.215786\end{array}$

O -0.621722 -0.763435 -0.362230

H $0.261288-1.279791-0.520379$

$\begin{array}{lllll}\text { O } & 2.606999 & 0.961783 & 0.249843\end{array}$

C $3.557721 \quad 0.437333-0.405832$

C $4.674609 \quad 1.397025-0.847332$

O $3.678710-0.774032-0.728845$

H $4.2663552 .121665-1.560836$

H $5.501336 \quad 0.856847-1.314060$

H $\quad 5.038187 \quad 1.968979 \quad 0.012953$

Zn -2.536914 -1.550599 -1.037090

H $2.296354-1.654972-0.749030$

O $1.448916-2.196916-0.726423$

H $\quad 1.486472-2.672557 \quad 0.127223$

O $\quad 1.523547-2.825748 \quad 2.025167$

H $\quad 0.981983-1.995872 \quad 2.002415$

H $2.428343-2.486517 \quad 2.055701$

\section{Structure 21}

C $0.922811-0.391743 \quad 0.297254$

N $1.582947-1.190179-0.793414$

C $2.821890-1.755939-0.834424$

N $3.529954-1.661679 \quad 0.366662$

C $3.148152-0.781001 \quad 1.382016$

C $1.961962-0.1630991 .380280$

H $1.129093-1.107105-1.693162$

O $3.303573-2.327485-1.812977$

H $\quad 4.492716-1.948245 \quad 0.282835$

H $3.888094-0.6328522 .162508$

H $1.677188 \quad 0.506204 \quad 2.181838$

N -0.169872 -1.206704 0.903466

H $\quad 0.176633-2.147031 \quad 1.088668$

H $-0.916011-1.292796 \quad 0.211670$

$\begin{array}{llll}\mathrm{O} & 0.389747 & 0.734673 & -0.227702\end{array}$

$\mathrm{H}-1.077283 \quad 1.262095 \quad 0.280144$

O $-3.694929-1.233341 \quad 0.387506$

C -4.128503 $-0.546983-0.541769$

C -5.181265 - $1.090372-1.495643$

O $-3.7705540 .675988-0.829606$

H -4.796582 -1.073042 -2.520115

H -6.066686 $-0.447125-1.473695$

H -5.453642 -2.108659 -1.216367

Zn $1.596025 \quad 2.373454-0.521302$

$\mathrm{H}-2.988251 \quad 1.052346-0.189118$

$\begin{array}{lll}\mathrm{O}-1.970526 & 1.582909 & 0.615783\end{array}$

$\mathrm{H}-2.066633 \quad 1.135386 \quad 1.490854$

$\begin{array}{lll}\mathrm{O}-2.200848 & -0.232727 & 2.648189\end{array}$

$\mathrm{H}-1.354232-0.577367 \quad 2.277527$

$\mathrm{H}-2.848365-0.640803 \quad 2.042634$

\section{Structure 22}

C -0.552953 $0.164375 \quad 0.722659$

N $-0.207645 \quad 1.378291-0.041724$

C - $1.010113 \quad 2.442114-0.324574$

$\begin{array}{lll}\mathrm{N}-2.208901 & 2.457323 & 0.398085\end{array}$

C $-2.506980 \quad 1.533312 \quad 1.384720$

$\begin{array}{lll}\text { C }-1.746318 & 0.450930 & 1.593159\end{array}$

H $0.684609 \quad 1.339479-0.538480$

O $-0.732787 \quad 3.356931-1.095844$

H -2.784929 $3.267660 \quad 0.239395$

$\mathrm{H}-3.402399 \quad 1.738443 \quad 1.962891$

$\mathrm{H}-1.984293-0.274501 \quad 2.359859$
N $0.598605-0.252133 \quad 1.525716$

$\begin{array}{llll}\mathrm{H} & 0.732641 & 0.448207 & 2.252968\end{array}$

H $1.419868-0.174790 \quad 0.913516$

O $-0.905927-0.895946-0.153201$

H -0.082038 -1.359991 - 0.553681

O $4.093490-0.527676 \quad 0.513031$

C $3.6350730 .330605-0.275736$

C $4.564316 \quad 1.457491-0.743754$

$\begin{array}{llll}\text { O } & 2.445647 & 0.364556 & -0.747239\end{array}$

H $4.088345 \quad 2.428387-0.572099$

H $4.722810 \quad 1.369391-1.824412$

H $5.527545 \quad 1.415911-0.230757$

Zn -2.898001-1.565524 -0.694239

H $1.732756-1.181509-1.207838$

O $1.192730-2.008836-1.161004$

H $1.618285-2.474276-0.400473$

O $2.439926-2.644742 \quad 1.203333$

H $1.728425-2.151706 \quad 1.645920$

H $\quad 3.101221-1.936613 \quad 0.994354$

\section{Structure 23}

C $0.812506-0.356633 \quad 0.213297$

N $1.551560-1.191578-0.799916$

C $2.691306-1.928228-0.659874$

N $\quad 3.122258-2.067030 \quad 0.660755$

C $2.627137-1.263387 \quad 1.690240$

C $1.564713-0.468310 \quad 1.526511$

H $1.337168-0.947253-1.757915$

O $3.297169-2.467360-1.586085$

H $4.034949-2.4871110 .740148$

H $\quad 3.170851-1.333956 \quad 2.627354$

H $\quad 1.190740 \quad 0.142515 \quad 2.338485$

N -0.538308 $-0.962167 \quad 0.424682$

H $-0.443649-1.974235 \quad 0.497085$

$\mathrm{H}-1.101782-0.764125-0.404527$

O $\quad 0.656459 \quad 0.903220-0.243898$

H $-0.825910 \quad 1.773721-0.020962$

O $-4.412659-0.514719 \quad 0.839772$

C - $4.327529-0.446706-0.468176$

C $-5.534949-1.055388-1.162857$

O $-3.398130 \quad 0.046828-1.106538$

H -5.400906 -1.022769 -2.244344

H $-6.437249-0.501522-0.884070$

H -5.675601 -2.088785 -0.831377

Zn $\quad 2.254539 \quad 2.164848-0.429863$

$\mathrm{H}-2.283536 \quad 1.654075-0.497881$

$\begin{array}{lll}\mathrm{O}-1.728179 & 2.170541 & 0.110688\end{array}$

H -2.187845 $1.232140 \quad 1.475517$

O $-2.383334 \quad 0.401811 \quad 1.994032$

$\mathrm{H}-1.657358-0.183007 \quad 1.639745$

H -3.554327 -0.110355 1.321371

Structure 24

$\begin{array}{lll}\text { C }-0.721399 & 0.215953 & 0.284484\end{array}$

$\mathrm{N}-0.961655 \quad 1.373750-0.616815$

C - $1.875656 \quad 2.384544-0.501595$

$\mathrm{N}-2.529084 \quad 2.425426 \quad 0.731818$

$\begin{array}{lll}\mathrm{C}-2.479352 & 1.368089 & 1.636762\end{array}$

$\begin{array}{lll}\mathrm{C}-1.652854 & 0.328307 & 1.473562\end{array}$

H $-0.5894991 .249815-1.549772$

O $-2.110319 \quad 3.215695-1.373904$

$\begin{array}{lll}\mathrm{H}-3.278515 & 3.098217 & 0.770520\end{array}$

H -3.164912 1.4461592 .474566

$\mathrm{H}-1.632862-0.490490 \quad 2.181427$

$\begin{array}{llll}\mathrm{N} & 0.723261 & 0.448264 & 0.815496\end{array}$

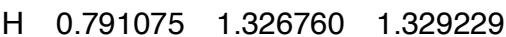

$\begin{array}{llll}H & 1.465927 & 0.465809 & 0.023961\end{array}$ 
O $-0.741106-0.947998-0.351609$

H $0.790951-1.948446-0.581278$

$\begin{array}{llll}O & 3.931462 & 0.395654 & 0.958538\end{array}$

C $3.797245 \quad 0.559180-0.274844$

C $5.024484 \quad 0.967657-1.098419$

O $2.7144850 .411510-0.948858$

H $4.785217 \quad 1.832408-1.725412$

H $5.2945750 .148563-1.774506$

H $\quad 5.873720 \quad 1.194427-0.450520$

Zn -2.447085 -2.083382 -0.576891

H $2.192292-1.489231-0.947329$

O $1.717660-2.273182-0.611295$

O $\quad 2.192159-1.520455 \quad 2.017122$

H $\quad 1.058680-0.336224 \quad 1.436540$

H $2.920365-0.896520 \quad 1.791404$

H $2.088350-2.0240421 .174762$

Structure 25

C $-1.869201 \quad 1.025268-0.292475$

$\mathrm{N}-3.2004891 .073232-0.243264$

C -3.913205 $-0.038902 \quad 0.107143$

$\mathrm{N}-3.163698-1.213474 \quad 0.400321$

C -1.803149-1.266403 0.346406

C -1.099584 $-0.159919 \quad 0.007985$

O $-5.138358-0.107721 \quad 0.187164$

H -3.715930 -2.021884 0.644803

$\mathrm{H}-1.316860-2.2112920 .572775$

H -0.019833 -0.171179 -0.045999

$\mathrm{N}-1.204923 \quad 2.144226-0.642241$

$\mathrm{H}-1.766167 \quad 2.944104-0.888284$

$\mathrm{H}-0.178120 \quad 2.146537-0.741394$

O $2.619084-0.723824-0.638192$

Zn $\quad 1.851627-2.560890 \quad 0.013805$

H $2.049002-0.037427-1.056804$

H $3.079223-0.095547-0.005437$

$\begin{array}{llll}\text { O } & 3.268733 & 1.453127 & 0.680639\end{array}$

C $2.342656 \quad 2.134389 \quad 0.173415$

C $2.128602 \quad 3.5596850 .698710$

O $\quad 1.550924 \quad 1.751647-0.753800$

H $2.2330824 .276269-0.123352$

H $2.843012 \quad 3.800021 \quad 1.488375$

H $\quad 1.107758 \quad 3.658671 \quad 1.083868$

\section{Structure 26}

C -0.280913 $0.583752 \quad 0.103464$

$\mathrm{N}-1.591258 \quad 0.902953-0.019962$

C - $2.001711 \quad 2.207700-0.081915$

$\mathrm{N}-0.9886613 .192553-0.012668$

C $\quad 0.342084 \quad 2.893063 \quad 0.121300$

$\begin{array}{llll}\text { C } & 0.745602 & 1.607269 & 0.186548\end{array}$

H -2.791598 -0.139060 -0.079258

O $-3.169364 \quad 2.575151-0.191416$

$\mathrm{H}-1.3201514 .143598-0.065881$

H $1.025571 \quad 3.7351220 .168992$

H $\quad 1.777057 \quad 1.289815 \quad 0.296410$

N $\quad 0.100921-0.682641 \quad 0.155567$

H $\quad 1.145747-0.884456 \quad 0.270708$

H $-0.619195-1.4044450 .110416$

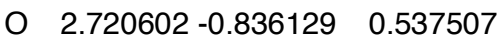

H $2.787591-0.876575 \quad 1.504024$

Zn $\quad 4.515166-0.743647-0.221549$

O -3.637044 -0.753787 -0.113352

C -3.352253 -2.032893 -0.028936

C $-4.597216-2.903968-0.070648$

O $-2.228093-2.517671 \quad 0.073349$

H -5.141029 -2.728254 -1.004095

H $-5.268297-2.6308310 .749683$
H -4.320055 -3.955598 0.007323

\section{Structure 27}

C - $0.445369 \quad 0.325424 \quad 0.800542$

$\mathrm{N}-0.033321 \quad 1.549524 \quad 0.122037$

C - $0.813910 \quad 2.407452-0.588049$

$\mathrm{N}-2.187367 \quad 2.183793-0.454229$

$\begin{array}{lll}\text { C }-2.719011 & 1.211556 & 0.382612\end{array}$

$\begin{array}{lll}\text { C - } 1.938721 & 0.321740 & 1.004423\end{array}$

$\begin{array}{llll}\mathrm{H} & 0.982526 & 1.648830 & 0.032303\end{array}$

O $-0.397777 \quad 3.338482-1.275854$

$\mathrm{H}-2.7694642 .851324-0.931663$

H $-3.798908 \quad 1.2275350 .491180$

H -2.355279 $-0.419244 \quad 1.673958$

$\begin{array}{llll}\mathrm{N} & 0.261008 & 0.288032 & 2.079485\end{array}$

H $\quad 0.247985-0.680575 \quad 2.394108$

H $1.240395 \quad 0.512192 \quad 1.879125$

O -0.136871 -0.822024-0.005117

H $\quad 0.905629-0.906423-0.140197$

$\begin{array}{llll}\mathrm{O} & 2.769416 & 0.968955 & 0.465566\end{array}$

$\mathrm{Zn}-1.428375-2.445140-0.447153$

C $3.106086-0.123203-0.061657$

C $4.587502-0.318014-0.416181$

O $2.342591-1.095801-0.356445$

H $4.728438-0.110248-1.483770$

H $4.892432-1.353912-0.241553$

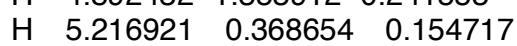

\section{Structure 28}

C -1.644568 $0.126683-0.524832$

$\mathrm{N}-2.238902-1.052635-0.307537$

C -3.578645 -1.121516-0.043815

$\mathrm{N}-4.288300 \quad 0.114525-0.017915$

C -3.684453 $1.325084-0.187374$

C - $2.358716 \quad 1.387137-0.449335$

O -4.214043 -2.147824 0.173872

H -5.270028 $0.033825 \quad 0.201167$

H -4.316905 2.203911 -0.106676

$\mathrm{H}-1.835895 \quad 2.325997-0.588167$

$\mathrm{N}-0.344974 \quad 0.160794-0.838404$

H $0.258234-0.676304-0.832807$

H $\quad 0.148605 \quad 1.041221-0.763473$

$\mathrm{H}-1.024832-2.2931390 .648008$

O $-0.210637-2.660102 \quad 1.052139$

H $\quad 0.415395-2.662864 \quad 0.299875$

O $1.561928-1.954854-0.965279$

C $2.780468-1.851936-0.640527$

O $3.336244-0.875990-0.056647$

C $3.671848-3.058799-0.968427$

H $4.728387-2.821658-0.826980$

H $3.402689-3.895285-0.312924$

H $3.491030-3.387086-1.996384$

H $\quad 0.684996-1.394490 \quad 1.959124$

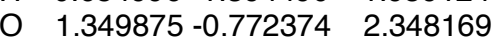

H $1.540094 \quad 0.746499 \quad 1.373395$

H $2.176631-1.050468 \quad 1.923318$

$\begin{array}{llll}\text { O } & 1.984289 & 1.259502 & 0.652928\end{array}$

$\begin{array}{llll}\mathrm{H} & 2.541838 & 0.529932 & 0.233604\end{array}$

Zn $1.319605 \quad 3.103812-0.060656$

\section{Structure 29}

C -1.012959-0.901999-0.639590

$\mathrm{N}-0.883558-2.221919-0.443578$

C - $1.970577-2.982967-0.102699$

$\mathrm{N}-3.206717-2.294413 \quad 0.034766$

C -3.322700 -0.937418 -0.067687

C $-2.250329-0.190238-0.414681$
O $-1.959343-4.194280 \quad 0.095583$

$\mathrm{H}-3.983226-2.873633 \quad 0.317867$

H -4.299065 -0.510496 0.139314

$\mathrm{H}-2.2855540 .892397-0.472769$

N $\quad 0.053015-0.198099-1.056777$

H $\quad 0.924370-0.712199-1.084276$

H $\quad 0.0929020 .814724-0.766270$

H $\quad 0.718855-2.251528 \quad 0.713040$

O $1.479490-1.909752 \quad 1.232731$

H $2.573322-1.564002 \quad 0.319013$

O $3.290216-1.343592-0.419990$

C $3.940741-0.214886-0.206432$

$\begin{array}{llll}\text { O } & 3.745179 & 0.562095 & 0.721661\end{array}$

C $4.9970940 .046061-1.265924$

H $\quad 5.530523 \quad 0.970047-1.041768$

H $5.697934-0.793222-1.309036$

H $4.521814 \quad 0.120593-2.249165$

H $1.196966-1.019413 \quad 1.592851$

$\begin{array}{llll}\mathrm{O} & 1.150058 & 0.623611 & 2.011639\end{array}$

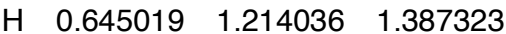

H $2.079887 \quad 0.758743 \quad 1.756925$

$\begin{array}{llll}\mathrm{O} & 0.002242 & 2.222435 & 0.107150\end{array}$

H $\quad 0.7782292 .766041-0.096805$

$\mathrm{Zn}-1.662717 \quad 3.286449-0.091842$

Structure $\mathbf{3 0}$

C $\quad 0.755994 \quad 0.859058-0.472534$

$\mathrm{N} \quad 0.544132 \quad 2.192862-0.188759$

C $1.5465323 .134921-0.060481$

N $2.8364512 .630202-0.276479$

C $3.1121301 .287994-0.411152$

C $2.119699 \quad 0.381684-0.513617$

$\begin{array}{llll}\text { O } & 1.365818 & 4.309924 & 0.211718\end{array}$

H $3.5732673 .309466-0.159173$

H $4.1613591 .013929-0.434528$

H $2.307684-0.685931-0.585575$

$\mathrm{N}-0.245426 \quad 0.051303-0.677235$

$\mathrm{H}-1.227748 \quad 0.394675-0.651315$

H -0.060549 -1.016647 -0.440792

$\mathrm{H}-0.395311 \quad 2.391452 \quad 0.286474$

$\begin{array}{lll}\mathrm{O}-1.692227 & 2.107425 & 1.220549\end{array}$

$\mathrm{H}-2.335380 \quad 1.7549820 .556816$

O $-2.972352 \quad 0.756402-0.747487$

C $-3.686383-0.251573-0.411655$

O -3.460335 -1.061083 0.516254

C -4.947803 $-0.466164-1.261481$

H -5.496172 -1.353678 -0.938441

H $-5.594706 \quad 0.414826-1.184708$

H -4.669750 -0.564704 -2.316375

$\mathrm{H}-1.456687 \quad 1.277167 \quad 1.705891$

O -1.321706 $-0.506896 \quad 2.143555$

$\mathrm{H}-0.636641-1.046328 \quad 1.704524$

$\mathrm{H}-2.137189-0.738549 \quad 1.631204$

$\begin{array}{lll}\text { O } & 0.287994-2.248643 & 0.256579\end{array}$ $\mathrm{H}-0.481428-2.836430 \quad 0.223779$

Zn $2.048154-3.098912-0.047476$

\section{Structure 31}

C -0.757453 -0.249197-0.841727

$\mathrm{N}-1.129153-1.407223-0.043171$

C $-2.380393-1.808726 \quad 0.316028$

$\mathrm{N}-3.423574-1.048011-0.225560$

C - $-3.213447 \quad 0.060834-1.024678$

C - $1.980494 \quad 0.475615-1.335937$

O -2.631727 $-2.763067 \quad 1.047153$

H - $-4.343092-1.321721 \quad 0.078880$

H $-4.107763 \quad 0.566575-1.374762$ 
$\mathrm{H}-1.824975 \quad 1.329465-1.982579$

N $0.064875-0.679780-1.955134$

H $\quad 0.921348-1.129208-1.612393$

H $\quad 0.372693 \quad 0.149334-2.460258$

H $-0.346769-1.956436 \quad 0.357043$

O $1.247858-2.558481 \quad 1.012212$

H $1.861086-2.281343 \quad 0.300672$

O $2.881075-1.368416-0.971049$

C $3.274333-0.265134-0.519506$

$\begin{array}{llll}O & 2.552964 & 0.611827 & 0.068522\end{array}$

C $4.7577910 .091260-0.661167$

H 4.861308 1.085176-1.107648

H $\quad 5.216104 \quad 0.138340 \quad 0.332955$

H $5.282866-0.650435-1.266263$

H $1.370457-1.868842 \quad 1.696407$

O $1.428806-0.148507 \quad 2.523661$

H $\quad 0.558511 \quad 0.0790892 .162005$

H $\quad 2.019677 \quad 0.207260 \quad 1.828737$

$\begin{array}{llll}\text { O }-0.044983 & 0.703343 & 0.008181\end{array}$

H $\quad 0.9630290 .580589-0.059130$

Zn -0.768783 $2.712525 \quad 0.452196$

\section{Structure TS1a}

$\begin{array}{llll}N & 1.618307 & 0.828000 & 0.044526\end{array}$

C $\quad 0.648863 \quad 1.7628520 .279510$

C -0.663585 $1.463736 \quad 0.148562$

H $\quad 0.996841 \quad 2.753765 \quad 0.551091$

C -0.999716 $0.121595-0.263703$

$\begin{array}{lll}\mathrm{H}-1.430973 & 2.211616 & 0.297048\end{array}$

H $2.593520 \quad 1.032156 \quad 0.208481$

N $0.008415-0.832363-0.456734$

$\mathrm{N}-2.129094-0.069553-1.015619$

C $1.339049-0.531188-0.210879$

H $-0.455191-1.492767 \quad 0.367491$

O $2.238808-1.350898-0.207264$

$\mathrm{H}-2.9480620 .448134-0.726435$

H -2.315043 -1.036439 -1.250554

O -1.321312 -1.014070 1.374835

H -2.212131 -1.381283 1.486162

\section{Structure TS1aw}

$\mathrm{N}-2.119206 \quad 0.005020 \quad 0.179382$ C - $1.804323-1.325557 \quad 0.239060$ C - $0.562221-1.762029-0.063039$ $\mathrm{H}-2.605425-1.992295 \quad 0.537557$

C $0.430763-0.779152-0.418537$ H $-0.301929-2.810384-0.014807$ H -3.023330 $0.347374 \quad 0.470519$ N $0.0049490 .508608-0.643955$ N $1.596695-1.134693-1.017001$ C -1.208273 $1.001562-0.194289$ O $-1.500394 \quad 2.180706-0.168474$ H $1.954148-2.037246-0.736056$ H $2.294489-0.379971-0.982344$

O $1.182334-0.198129 \quad 1.539600$

H $1.747315-0.880152 \quad 1.929468$

H $\quad 0.813609 \quad 1.179286-0.615827$

$\begin{array}{llll}H & 1.927383 & 0.599616 & 0.900384\end{array}$

$\begin{array}{llll}\mathrm{O} & 2.427183 & 1.202136 & 0.026270\end{array}$

H $2.808016 \quad 2.034580 \quad 0.323782$

\section{Structure TS1b}

C -0.599806 $1.340352-0.260059$ C -0.824664 -0.096467-0.156903

$\mathrm{N} \quad 0.153846-0.987365-0.187115$

C $1.447628-0.597837 \quad 0.017244$

O $2.416901-1.335310 \quad 0.112156$
$\begin{array}{llll}\mathrm{N} & 1.673678 & 0.816052 & 0.068169\end{array}$

H $2.642276 \quad 1.0881520 .153593$

C $0.687887 \quad 1.736559-0.111523$

H $0.987638 \quad 2.779891-0.122921$

$\mathrm{N}-2.064548-0.620792-0.887322$

$\mathrm{H}-1.410566 \quad 2.050809-0.363481$

H - $2.328742-0.084328-1.711249$

H - $1.868829-1.595557-1.124104$

O $-1.927158-0.292690 \quad 1.314251$

$\begin{array}{lll}\mathrm{H}-1.909324 & 0.539297 & 1.806897\end{array}$

H -2.637493 $-0.505172 \quad 0.061322$

\section{Structure TS1 bw}

$\mathrm{N}-2.184169 \quad 0.705078-0.189327$

C -1.283965 $1.720258-0.050750$

C $\quad 0.015307 \quad 1.453588 \quad 0.211101$

H -1.666799 2.729559-0.163939

$\begin{array}{llll}\text { C } & 0.366369 & 0.037952 & 0.281093\end{array}$

$\begin{array}{llll}H & 0.763880 & 2.233176 & 0.268448\end{array}$

H -3.160904 $0.885117-0.369669$

O $1.304969-0.187360-1.265711$

$\mathrm{N}-0.552758-0.930363 \quad 0.339653$

N $1.509243-0.278665 \quad 1.220507$

C -1.851301-0.669379 -0.004490

O $-2.741426-1.501052-0.117831$

H $2.551812-0.185648-0.954655$

$\begin{array}{llll}H & 1.437173 & 0.319098 & 2.044270\end{array}$

H $1.319171-1.239725 \quad 1.517545$

H $2.579285-0.2001430 .664175$

O $3.476017-0.100884-0.281617$

H $4.011619-0.899998-0.368286$

H $0.963608-1.053934-1.534182$

Structure TS2a

$\begin{array}{lll}\mathrm{N}-1.603813 & 0.852717 & 0.224309\end{array}$

C $-0.587282 \quad 1.764311-0.024102$

C $0.672696 \quad 1.367268-0.259663$

H -0.887722 $2.806597-0.021174$

C $\quad 1.023639-0.080342-0.250817$

H $1.4628882 .071958-0.483267$

$\mathrm{H}-2.560193 \quad 1.157903 \quad 0.324165$

O $2.017006-0.535236-1.010613$

$\mathrm{N}-0.160414-0.895773-0.250383$

N $1.911647-0.434381 \quad 1.191757$

C -1.460753 $-0.516683 \quad 0.001916$

H $-0.032612-1.849966-0.560916$

O -2.410021-1.284509 0.018750

H $2.514740-0.769898 \quad 0.168533$

H $\quad 2.243744 \quad 0.377234 \quad 1.711452$

H $1.481541-1.1011341 .832326$

\section{Structure TS2aw}

$\mathrm{N}-2.044792 \quad 0.911254 \quad 0.169816$

C -0.975286 $1.762771-0.087655$

C $0.269347 \quad 1.301628-0.263725$

$\mathrm{H}-1.2266602 .816615-0.144358$

C $0.564167-0.174107-0.187794$

H $1.099751 \quad 1.959440-0.485934$

$\mathrm{H}-2.990206 \quad 1.262776 \quad 0.184781$

O $1.401770-0.682778-1.086320$

$\mathrm{N}-0.665778-0.936494-0.113231$

N $1.332100-0.359808 \quad 1.256446$

C -1.956215 -0.470379 0.013776

H - $0.591657-1.888254-0.448780$

O -2.943394 -1.189892 -0.001553

H $2.501681-0.290666-0.744109$

H $\quad 0.931004 \quad 0.2301751 .983555$
H $\quad 1.248950-1.336118 \quad 1.539386$

H $\quad 2.439347-0.105185 \quad 0.914617$

$\begin{array}{llll}\text { O } & 3.381661 & 0.127523 & -0.017784\end{array}$

H $4.104698-0.5117590 .007261$

\section{Structure TS2b}

C -1.223366 -0.229941 -0.000090

$\mathrm{N}-0.096137-0.979950-0.000460$

C $1.180445-0.478112-0.000110$

$\begin{array}{llll}\mathrm{N} & 1.182036 & 0.951644 & 0.000103\end{array}$

$\begin{array}{llll}\text { C } & 0.067199 & 1.731027 & 0.000231\end{array}$

C -1.188355 $1.195289 \quad 0.000124$

O $2.226243-1.094719-0.000042$

$\mathrm{H}-2.084026 \quad 1.798547 \quad 0.000314$

H $\quad 0.239910 \quad 2.802352 \quad 0.000461$

H $2.104330 \quad 1.365886 \quad 0.000286$

O -2.197414 -1.061757 0.000116

H -1.107673 -1.826418 -0.000100

\section{Structure TS2bw}

C $-0.549901-0.863139-0.000914$ $\mathrm{N}-0.339492 \quad 0.477394-0.007951$

C $0.909740 \quad 1.053025-0.003437$

$\begin{array}{llll}\mathrm{N} & 1.980417 & 0.127940 & 0.001503\end{array}$

C $1.811250-1.2240120 .008775$

C $\quad 0.568755-1.770247 \quad 0.010751$

O $1.134055 \quad 2.250763-0.000181$

H $\quad 0.400345-2.837574 \quad 0.015202$

H $\quad 2.717650-1.820316 \quad 0.012601$

H $2.900907 \quad 0.544742 \quad 0.000884$

O -1.757714 -1.296082 -0.005663

$\mathrm{H}-2.471283-0.275000-0.036285$

O $-2.725353 \quad 0.890002-0.086836$

$\mathrm{H}-1.569167 \quad 1.035752-0.046563$

$\begin{array}{lll}\mathrm{H}-3.111893 & 1.183827 & 0.749686\end{array}$

\section{Structure TS3}

N $2.572496-0.378230 \quad 0.877209$

C $\quad 1.582332 \quad 0.038014 \quad 1.755319$

$\begin{array}{llll}\text { C } & 0.434272 & 0.580942 & 1.326571\end{array}$

H $1.795521-0.122146 \quad 2.806525$

C $0.1755530 .781405-0.143450$

$\mathrm{H}-0.344186 \quad 0.882892 \quad 2.015131$

H $3.374235-0.892401 \quad 1.210427$

O -1.060853 $0.425193-0.586202$

N $1.2078240 .143279-0.933050$

$\mathrm{N} \quad 0.264125 \quad 2.348471-0.392756$

C $2.364587-0.473615-0.496345$

H $0.933749-0.110524-1.873733$

O $3.145954-1.042936-1.239630$

$\mathrm{H}-1.827690 \quad 1.375628-0.256214$

H $\quad \begin{array}{lll}1.003890 & 2.762900 & 0.173359\end{array}$

H $0.4897382 .504776-1.375943$

$\mathrm{H}-0.9153812 .695184-0.144294$

$\begin{array}{lll}\mathrm{O}-2.132836 & 2.458739 & 0.068867\end{array}$

$\mathrm{H}-2.718240 \quad 2.847141-0.594358$

Zn -1.902045 -1.567551 0.019414

\section{Structure TS4}

C -0.461295 $0.241598 \quad 0.812642$

N $0.020251 \quad 1.180126-0.175234$

C - $0.558496 \quad 2.353068-0.558761$

$\mathrm{N}-1.735986 \quad 2.686020 \quad 0.128609$

C -2.278462 $1.899560 \quad 1.127174$

$\begin{array}{lll}\text { C }-1.698320 & 0.753597 & 1.504649\end{array}$

H $\quad 0.9300660 .923339-0.631132$

O $-0.123477 \quad 3.110731-1.420616$ 
H -2.173789 $3.537728-0.181309$

$\mathrm{H}-3.197905 \quad 2.268874 \quad 1.571624$ $\mathrm{H}-2.129036 \quad 0.118131 \quad 2.268551$

$\begin{array}{llll}\text { N } & 0.619767 & 0.001678 & 1.845677\end{array}$

H $\quad 0.651179 \quad 0.783081 \quad 2.497342$

H $1.607105-0.110475 \quad 1.390812$

O -0.691149 -1.025714 0.239844

H $-0.590176-1.752424 \quad 1.092344$

O -0.279681 -2.255559 2.293096

H $\quad 0.429357-2.894313 \quad 2.137028$

H $\quad 0.306309-1.0646812 .306831$

O $2.489531 \quad 0.404317-1.132751$

C $3.290586-0.085231-0.300327$

C $4.701468-0.441717-0.798042$

O $3.066716-0.341154 \quad 0.926307$

H $4.817576-1.531843-0.809081$

H $5.455244-0.045945-0.109270$

H $4.871489-0.054196-1.804907$

Zn -1.899135 -1.823942 -1.343108

\section{Structure TS5}

$\begin{array}{llll}C & 0.744402 & 0.099784 & 0.368813\end{array}$

N $\quad 0.771913-1.353178 \quad 0.039991$

C $1.830962-2.138404-0.287598$

N $3.082457-1.547164-0.078819$

C $3.231329-0.190179 \quad 0.210527$

$\begin{array}{llll}\text { C } & 2.175688 & 0.593904 & 0.449374\end{array}$

H -0.145535 - $1.748237-0.159494$

O $1.757252-3.296697-0.705376$

H $3.849733-2.086214-0.446320$

H $4.256895 \quad 0.1680910 .232925$

H $2.291823 \quad 1.6521720 .656125$

O $-0.009210 \quad 0.810837-0.498457$

$\begin{array}{llll}\mathrm{N} & 0.045823 & 0.173502 & 1.693229\end{array}$

H $0.021031 \quad 1.161045 \quad 1.952395$

O $-2.217607-2.020298-0.255648$

C -3.007537 -1.132096 0.021763

O -2.695694 $0.046288 \quad 0.544350$

C -4.503103-1.260165 -0.205047

H -5.047235 -1.032061 0.716345

H -4.820772 -0.532546 -0.958805

H - $4.739422-2.268951-0.544198$

H $0.569182-0.330261 \quad 2.409519$

$\mathrm{H}-1.708945 \quad 0.140684 \quad 0.637357$

Zn $\quad 0.022117 \quad 2.793535-0.403420$

\section{Structure TS6}

$\begin{array}{lll}\text { C }-0.728685 & 0.296959 & 0.627462\end{array}$

$\mathrm{N}-0.046151 \quad 0.966227-0.463927$

C - $-0.236553 \quad 2.244658-0.896437$

$\mathrm{N}-1.288688 \quad 2.933567-0.262467$

$\begin{array}{lll}\mathrm{C}-2.137269 & 2.335949 & 0.653217\end{array}$

$\begin{array}{lll}C-1.895427 & 1.104968 & 1.124710\end{array}$

H $0.8212660 .484899-0.794260$

O $\quad 0.406238 \quad 2.808161-1.777344$

$\mathrm{H}-1.515455 \quad 3.815261-0.693518$

$\mathrm{H}-2.997140 \quad 2.928545 \quad 0.953152$

$\mathrm{H}-2.563887 \quad 0.619412 \quad 1.824301$

$\begin{array}{llll}\mathrm{N} & 0.461113 & 0.430863 & 1.920539\end{array}$

H $1.4650390 .106140 \quad 1.567700$

O $2.858194-0.301499 \quad 1.240362$

C $3.124228-0.4969420 .006920$

H $\quad 0.521402 \quad 1.3729542 .305823$

O -0.909115 $-0.976952 \quad 0.514830$

O $2.381922-0.278418-0.976581$

C $4.517228-1.087093-0.267252$

H $4.754329-1.048361-1.332659$
H $\quad 4.538459-2.131688 \quad 0.065024$

H $\quad 5.277123-0.549723 \quad 0.309587$

H $\quad 0.137723-0.206524 \quad 2.645610$

Zn -1.936061 -2.403562 -0.490388

\section{Structure TS7}

$\begin{array}{lll}\mathrm{C}-0.603733 & 0.210192 & 0.788750\end{array}$

$\begin{array}{lll}\mathrm{N}-0.261744 & 1.473147 & 0.070113\end{array}$

C -1.098984 2.379949 -0.499951

$\mathrm{N}-2.435466 \quad 2.234033-0.119323$

C -2.875294 $1.239145 \quad 0.742598$

$\begin{array}{lll}C-2.053885 & 0.289622 & 1.207944\end{array}$

H $0.7113221 .538984-0.213290$

O $-0.756800 \quad 3.296346-1.248740$

$\mathrm{H}-3.0700942 .891322-0.541160$

$\mathrm{H}-3.930972 \quad 1.2764790 .993928$

H -2.408051 -0.477524 1.884413

$\begin{array}{llll}\mathrm{N} & 0.256095 & 0.093390 & 1.981539\end{array}$

$\begin{array}{llll}\mathrm{H} & 0.024169 & 0.869967 & 2.598904\end{array}$

$\begin{array}{llll}H & 1.214380 & 0.271401 & 1.661803\end{array}$

O $-0.393852-0.884973-0.036589$

$\begin{array}{llll}\mathrm{O} & 2.744120 & 0.874641 & 0.436380\end{array}$

C $3.5802090 .150697-0.128551$

C $4.763263 \quad 0.782199-0.857894$

O $3.562137-1.140680-0.184236$

H $\quad 4.617498 \quad 0.675030-1.938437$

H $\quad 5.690144 \quad 0.260049-0.602621$

H $4.842101 \quad 1.842399-0.611755$

Zn -1.968960 -2.022291 -0.779539

H $2.566481-1.6259010 .257431$

O $1.538063-2.169702 \quad 0.654594$

H $1.454500-2.008722 \quad 1.605685$

H $\quad 0.626342-1.564628 \quad 0.276297$

\section{Structure TS8}

C $0.650864-0.231651 \quad 0.390026$

N $1.054227-1.309244-0.560234$

C $2.179981-2.075335-0.582417$

N $3.009340-1.9052690 .531280$

C $2.853975-0.838640 \quad 1.415772$

C $1.778628-0.043098 \quad 1.389259$

H $\quad 0.504779-1.334160-1.409183$

O $2.473487-2.872542-1.471939$

H $3.901462-2.364918 \quad 0.439108$

H $3.670685-0.700761 \quad 2.117928$

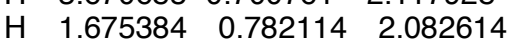

N -0.558071 $-0.741598 \quad 1.163139$

H $-0.298261-1.486872 \quad 1.806349$

H -1.298040 -1.091984 0.517188

O $0.281280 \quad 0.882181-0.262740$

O -2.922435 -1.356718 -0.252339

C $-3.866840-0.540149-0.189881$

C -5.178356 -0.893126 -0.901774

$\begin{array}{lll}\mathrm{O}-3.881118 & 0.594964 & 0.403860\end{array}$

H -5.325476 -0.212310 - 1.747711

H $-6.025523-0.749499-0.223549$

H -5.158040 -1.921829 -1.267222

Zn $\quad 1.510505 \quad 2.421075-0.727441$

$\mathrm{H}-2.743026 \quad 1.024791 \quad 0.990421$

$\begin{array}{lll}\mathrm{O}-1.805043 & 1.358163 & 1.475806\end{array}$

$\mathrm{H}-1.226998 \quad 1.671321 \quad 0.744182$

$\mathrm{H}-1.209445 \quad 0.328234 \quad 1.566712$

\section{Structure TS9}

C $-0.740753 \quad 0.144342 \quad 0.419477$

$\mathrm{N}-0.357670 \quad 1.241715-0.428950$

C - $0.934901 \quad 2.483453-0.482559$
$\mathrm{N}-2.125221 \quad 2.597860 \quad 0.246946$

C -2.672015 $1.560082 \quad 0.980832$

$\begin{array}{lll}C-2.039651 & 0.383325 & 1.104631\end{array}$

H $0.614723 \quad 1.193058-0.755933$

O $-0.508038 \quad 3.434019-1.127043$

$\mathrm{H}-2.607338 \quad 3.473701 \quad 0.124581$

H -3.636312 $1.765038 \quad 1.435343$

H -2.460093 -0.435677 1.671987

$\begin{array}{llll}\mathrm{N} & 0.536280 & 0.405088 & 1.839480\end{array}$

H $\quad 0.280999 \quad 1.072733 \quad 2.562194$

H $1.373405 \quad 0.717105 \quad 1.285974$

O -0.462627-1.033528 -0.001050

O $\quad 2.426637 \quad 0.995793-0.046591$

C $3.3387210 .126161-0.246489$

C $4.251650 \quad 0.402390-1.454006$

O $3.562635-0.907279 \quad 0.425539$

H $3.6729340 .289507-2.378150$

H $5.097130-0.289244-1.476341$

H $4.610481 \quad 1.436542-1.426519$

$\mathrm{Zn}-1.903614-2.246796-0.920860$

H $\quad 2.336324-1.764011 \quad 1.375157$

O $\quad 1.551742-2.199410 \quad 1.796298$

H $\quad 0.894594-2.157405 \quad 1.080624$

H $\quad 0.776767-0.5253792 .216008$

\section{Structure TS10}

$\begin{array}{lll}\text { C }-0.670726 & 0.153905 & 0.668053\end{array}$

$\mathrm{N}-0.522371 \quad 1.538554 \quad 0.134817$

C - $1.485404 \quad 2.460372-0.135105$

$\mathrm{N}-2.743844 \quad 2.133155 \quad 0.376206$

$\begin{array}{lll}\text { C }-3.004181 & 0.950229 & 1.053245\end{array}$

$\begin{array}{lll}C-2.065491 & 0.012736 & 1.233850\end{array}$

H $\quad 0.401250 \quad 1.747743-0.232335$

O -1.306954 $3.522020-0.732181$

$\mathrm{H}-3.475200 \quad 2.785642 \quad 0.147202$

$\mathrm{H}-4.023623 \quad 0.834123 \quad 1.408272$

H -2.284956 -0.905245 1.763734

N $0.331721-0.005808 \quad 1.759372$

H $\quad 0.101015 \quad 0.673451 \quad 2.483679$

H $1.228690 \quad 0.309071 \quad 1.356605$

O $-0.433125-0.783373-0.306784$

H $\quad 0.752121-1.414385-0.329048$

$\begin{array}{lllll}O & 2.550493 & 1.192247 & 0.279987\end{array}$

C $3.4656470 .690463-0.408208$

C $4.5485591 .611280-0.975223$

O $3.601233-0.545029-0.719023$

H $\quad 4.374587 \quad 1.748386-2.048498$

H $5.5353821 .152516-0.863178$

H $\quad 4.5225242 .586978-0.486158$

$\mathrm{Zn}-1.953912-1.817709-1.269404$

H $2.574026-1.314367-0.447508$

O $1.691136-1.991713-0.281448$

H $\quad 1.702074-2.339080 \quad 0.643688$

O $1.391151-2.457261 \quad 2.395354$

H $\quad 0.854124-1.616183 \quad 2.291934$

H $2.214914-2.147736 \quad 2.794056$

\section{Structure TS11}

C -0.749062 $0.231420 \quad 0.461845$

$\mathrm{N}-0.962818 \quad 1.418430-0.424024$

C - $1.992251 \quad 2.310642-0.455372$

$\begin{array}{lll}\mathrm{N}-2.945105 & 2.115642 & 0.548036\end{array}$

C -2.984962 $0.956509 \quad 1.319496$

$\begin{array}{lll}C-2.009720 & 0.039865 & 1.280444\end{array}$

H -0.329259 $1.480674-1.209694$

O $-2.102510 \quad 3.230021-1.265293$

$\mathrm{H}-3.767444 \quad 2.688158 \quad 0.440344$ 
$\mathrm{H}-3.869414 \quad 0.844732 \quad 1.938646$ $\mathrm{H}-2.040849-0.837003 \quad 1.913150$ $\begin{array}{llll}\mathrm{N} & 0.362527 & 0.478015 & 1.399114\end{array}$

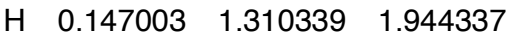
H $\quad 1.231146 \quad 0.681755 \quad 0.892712$ O $-0.464804-0.859509-0.324086$

H $\quad 0.713049-1.390062-0.294777$

$\begin{array}{llll}\text { O } & 3.385599 & 0.704639 & 0.742974\end{array}$

C $3.7976690 .535782-0.437292$

C $4.824443 \quad 1.544850-0.962053$

O $3.441840-0.379442-1.243584$

H $\quad 5.164910 \quad 1.283406-1.965784$

H $5.677390 \quad 1.589242-0.277006$

H $4.375172 \quad 2.543833-0.976964$

Zn -2.115594 -2.080129 -0.787394

H $2.453253-1.271314-0.718436$

O $1.722898-1.909102-0.275619$

H $\quad 1.981254-1.9404850 .688515$

O $2.367378-1.384606 \quad 2.262350$

H $1.507952-0.915432 \quad 2.315138$

H $2.921504-0.6789851 .853430$

\section{Structure TS12}

C $0.762158-0.311276 \quad 0.269073$

N $1.370998-1.301777-0.678809$

C $2.480093-2.082013-0.532893$

N $3.034784-2.050130 \quad 0.748895$

C $2.685350-1.075111 \quad 1.683180$

C $1.651399-0.2470591 .496672$

H $1.058044-1.199121-1.635138$

O $2.964752-2.786244-1.417636$

H $\quad 3.926071-2.5166790 .810545$

H $3.319286-1.0344612 .563469$

H $\quad 1.390129 \quad 0.498142 \quad 2.237093$

N -0.569220 $-0.822428 \quad 0.712155$

H $-0.464796-1.784536 \quad 1.030294$

$\mathrm{H}-1.202012-0.837034-0.094167$

O $\quad 0.593660 \quad 0.879467-0.359215$

H $-0.8022991 .583739-0.350799$

O $-4.313729-0.235481 \quad 0.891899$

C -4.154815 -0.502188 -0.338489

C -5.249473 -1.345342 - 1.000267

O $-3.200457-0.132666-1.081358$

H $-4.799947-2.088191-1.665233$

H -5.875700 -0.693163 -1.620148

H -5.880227 -1.832875 -0.253903

$\mathrm{Zn} \quad 2.226690 \quad 2.127916-0.548864$

$\mathrm{H}-2.318051 \quad 1.273544-0.736973$

O $-1.753800 \quad 1.950620-0.262138$

$\begin{array}{lll}\mathrm{H}-2.049400 & 1.592367 & 0.897789\end{array}$

$\begin{array}{lll}\mathrm{O}-2.315657 & 0.957776 & 1.864502\end{array}$

$\begin{array}{lll}\mathrm{H}-1.614418 & 0.253501 & 1.727731\end{array}$

$\mathrm{H}-3.189737 \quad 0.477796 \quad 1.527584$

Structure TS13

C - $0.747112 \quad 0.273955 \quad 0.174444$

$\mathrm{N}-1.3041391 .302354-0.756899$

C $-2.336919 \quad 2.176506-0.567849$

$\mathrm{N}-2.811419 \quad 2.223812 \quad 0.745039$

C $-2.497339 \quad 1.239533 \quad 1.682067$

$\begin{array}{lll}C-1.554567 & 0.317209 & 1.458334\end{array}$

$\mathrm{H}-1.074335 \quad 1.134429-1.727780$

O $-2.815835 \quad 2.893174-1.443883$

$\mathrm{H}-3.651734 \quad 2.771255 \quad 0.846658$

$\mathrm{H}-3.081068 \quad 1.2727712 .596588$

$\mathrm{H}-1.320399-0.437752 \quad 2.197973$

$\begin{array}{llll}\mathrm{N} & 0.663322 & 0.727797 & 0.533168\end{array}$
$\begin{array}{llll}\mathrm{H} & 0.649237 & 1.693519 & 0.860219\end{array}$ H $1.2811020 .680619-0.292383$ O $-0.669682-0.924399-0.415122$

H $\quad 0.852032-1.878439-0.439910$

$\begin{array}{lllll}O & 4.182119 & 0.307030 & 0.963480\end{array}$

C $4.0857320 .489726-0.289308$

C $5.295258 \quad 1.136403-0.975007$

O $3.1103830 .176154-1.029440$

H $4.9626901 .837219-1.745792$

H $\quad 5.878763 \quad 0.354828-1.475857$

H $\quad 5.939305 \quad 1.640126-0.250647$

Zn -2.329254 -2.143274 -0.443056

H $2.312165-1.526009-0.760695$

O $1.770977-2.224409-0.333764$

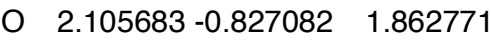

H $1.332332-0.067123 \quad 1.345475$

H $3.008204-0.382760 \quad 1.565497$

H $2.015497-1.5759521 .194766$

Structure TS14

C-1.065112 0.2120890 .198718 $\mathrm{N}-1.500522 \quad 1.092467-0.840314$

C - $2.051738 \quad 2.345385-0.709155$

$\begin{array}{lll}\mathrm{N}-2.334551 & 2.688807 & 0.610663\end{array}$

$\begin{array}{lll}\mathrm{C}-2.191476 & 1.806831 & 1.675817\end{array}$

$\begin{array}{lll}C-1.591563 & 0.617416 & 1.522861\end{array}$

$\mathrm{H}-1.279224 \quad 0.803374-1.783822$

O $-2.304614 \quad 3.094433-1.643636$

H $-2.824902 \quad 3.562796 \quad 0.717647$

$\mathrm{H}-2.586498 \quad 2.155443 \quad 2.623784$

$\mathrm{H}-1.451095-0.069418 \quad 2.345919$

$\begin{array}{llll}\mathrm{N} & 0.826057 & 0.876923 & 0.466395\end{array}$

$\begin{array}{llll}\mathrm{H} & 0.886331 & 1.892956 & 0.496068\end{array}$

H $1.465353 \quad 0.527805-0.272707$

O -0.918286-1.007025 -0.123153

H $\quad 0.767613-1.967240 \quad 0.288224$

$\begin{array}{llll}\text { O } & 4.265430 & 0.459066 & 0.642669\end{array}$

C $4.0265430 .218970-0.564540$

C $5.1284580 .558418-1.586539$

O $2.964250-0.282543-1.064003$

H $4.716559 \quad 1.183662-2.386245$

H $\quad 5.483708-0.365158-2.057985$

H $\quad 5.969590 \quad 1.068087-1.110314$

$\mathrm{Zn}-2.558885-2.330931-0.482359$

H $2.204918-1.663916-0.146689$

O $1.682258-2.2610620 .438806$

O $\quad 2.367626-0.4418992 .457436$

$\begin{array}{llll}H & 1.237528 & 0.498057 & 1.343682\end{array}$

H $3.120314-0.096461 \quad 1.916336$

H $2.135477-1.251874 \quad 1.951763$

\section{Structure TS15}

C -0.164154 $0.524150 \quad 1.002713$

$\begin{array}{llll}\mathrm{N} & 0.634925 & 1.251684 & 0.142652\end{array}$

C $0.189780 \quad 2.312703-0.611029$

$\mathrm{N}-1.085097 \quad 2.776759-0.265927$

C-1.842742 $2.238609 \quad 0.753378$

C $-1.418993 \quad 1.150956 \quad 1.415588$

H $1.613307 \quad 0.858136-0.100318$

O $0.837276 \quad 2.871052-1.485255$

$\mathrm{H}-1.401893 \quad 3.567169-0.804360$

$\begin{array}{lll}\mathrm{H}-2.779880 & 2.740314 & 0.968102\end{array}$

$\begin{array}{lll}\mathrm{H}-2.005681 & 0.693910 & 2.200511\end{array}$

N $0.494726-0.294636 \quad 1.868633$

H -0.137586 -0.937745 2.327508

H $\quad 1.332610-0.790630 \quad 1.468571$

O $-1.075781-0.809182-0.225263$
H -0.300970 -1.304044-0.539585

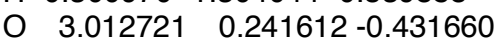

Zn -2.744825 -1.800784 -0.550243

C $3.286952-0.925436-0.024825$

C $4.605936-1.518022-0.546405$

O $2.597658-1.640679 \quad 0.753255$

H $4.580467-1.563742-1.640546$

H $4.778755-2.517344-0.140605$

H $\quad 5.438083-0.859340-0.274627$

\section{Structure TS16}

C -1.004854 -0.856308 -0.683553

$\mathrm{N}-0.955261-2.163157-0.379973$

C -2.095707-2.844381-0.038736

$\mathrm{N}-3.299244-2.089328-0.020420$

C -3.333766 $-0.736126-0.202215$

C -2.208906 $-0.069283-0.547425$

O $-2.152593-4.033672 \quad 0.256004$

H -4.116584 -2.606038 0.269112

$\mathrm{H}-4.291010-0.244912-0.058314$

$\mathrm{H}-2.181815 \quad 1.010267-0.650840$

N $0.110597-0.242082-1.109255$

H $0.968468-0.787439-1.103540$

H $\quad 0.197846 \quad 0.769689-0.857101$

H $\quad 0.489174-2.176467 \quad 0.788610$

O $1.265603-1.853138 \quad 1.316948$

H $2.060638-1.738267 \quad 0.611559$

O $2.999402-1.430021-0.410526$

C $3.767782-0.416406-0.237606$

$\begin{array}{llll}\text { O } & 3.666111 & 0.460850 & 0.649197\end{array}$

C $4.914861-0.303778-1.243378$

H $\quad 5.5384040 .565928-1.028827$

H $5.521372-1.214833-1.213930$

H $4.506984-0.224837-2.256654$

H $1.129456-0.750036 \quad 1.619278$

$\begin{array}{llll}\mathrm{O} & 1.295409 & 0.506871 & 1.870303\end{array}$

H $\quad 0.773555 \quad 1.145684 \quad 1.272202$

H $2.234990 \quad 0.582135 \quad 1.533898$

$\begin{array}{llll}\mathrm{O} & 0.189626 & 2.161123 & 0.144037\end{array}$

H $\quad 0.9840512 .673002-0.072487$

$\mathrm{Zn}-1.438436 \quad 3.310656-0.039189$

\section{Structure TS17}

C $0.705393 \quad 0.906318-0.589467$

$\mathrm{N} \quad 0.349134 \quad 2.172123-0.222130$

C $1.2725523 .171014-0.005990$

N $2.616840 \quad 2.794724-0.205439$

C $3.011025 \quad 1.497374-0.412827$

C $2.0968750 .524671-0.615856$

$\begin{array}{llll}\text { O } & 1.003231 & 4.312740 & 0.337369\end{array}$

H $3.286895 \quad 3.524263-0.012014$

H $4.079418 \quad 1.308312-0.407676$

H $2.370622-0.519931-0.730148$

$\mathrm{N}-0.226950 \quad 0.022249-0.888520$

$\mathrm{H}-1.2138720 .303809-0.841872$

H $0.007504-1.003684-0.642005$

$\mathrm{H}-0.706156 \quad 2.159204 \quad 0.434301$

$\begin{array}{lll}\mathrm{O}-1.678699 & 1.826388 & 1.169428\end{array}$

$\mathrm{H}-2.364750 \quad 1.449317 \quad 0.515349$

O $-3.109214 \quad 0.664595-0.633714$

C -3.735703 -0.410178 -0.316679

O -3.486729-1.171411 0.642559

C $-4.908726-0.765657-1.239016$

$\mathrm{H}-5.377417-1.704172-0.936117$

$\mathrm{H}-5.6492280 .041380-1.216271$

H -4.554997 $-0.843047-2.272626$

$\mathrm{H}-1.3836330 .981861 \quad 1.633985$ 
O -1.181995 -0.649023 2.037007

$\mathrm{H}-0.517815-1.165563 \quad 1.527844$ H -2.043258 -0.911681 1.624961 O $\quad 0.435987-2.230518 \quad 0.195976$ $\mathrm{H}-0.239565-2.920505 \quad 0.117588$

Zn $\quad 2.287465-2.906222-0.016422$

\section{Structure TS18}

C $-0.709109-0.721942-1.114383$ N $-0.857048-1.690407-0.145067$ C -2.042825 -1.973219 0.504644 $\mathrm{N}-3.167538-1.367938-0.058772$ C -3.113605 $-0.530268-1.152102$ C -1.935030 -0.202297-1.705998 O $-2.141341-2.723790 \quad 1.462124$ $\mathrm{H}-4.034357-1.567608 \quad 0.415238$ $\mathrm{H}-4.063405-0.149404-1.509002$ $\mathrm{H}-1.871603 \quad 0.477256-2.544127$ N $0.421415-0.763865-1.861023$ H $1.334960-0.973252-1.394664$ H $\quad 0.5120090 .037848-2.471599$ H $0.018334-2.0486320 .337221$ O $1.450288-2.295572 \quad 1.123818$ H $2.112468-2.0163840 .454888$ O $2.978372-1.080761-0.867116$ C $3.384970 \quad 0.043589-0.418567$ O $2.666713 \quad 0.986974-0.003744$ C $4.9092960 .222952-0.365575$ H $5.370984-0.173731-1.274674$ H $\quad 5.175533 \quad 1.273324-0.229090$ H $\quad 5.308629-0.3533540 .477474$ H $1.375863-1.497254 \quad 1.705426$ $\begin{array}{llll}\mathrm{O} & 1.146383 & 0.182761 & 2.275148\end{array}$ H $\quad 0.380922 \quad 0.415910 \quad 1.700834$ H $1.866458 \quad 0.579907 \quad 1.749286$ $\begin{array}{llll}\text { O } & -0.353329 & 0.991237 & 0.054342\end{array}$ H $0.601179 \quad 1.113176-0.128686$ Zn-1.260155 $2.737266 \quad 0.344028$

\section{MP2 level}

Structure 16

C - $0.445052 \quad 0.159432 \quad 0.958831$ $\begin{array}{llll}\mathrm{N} & 0.051703 & 1.393636 & 0.354393\end{array}$ C - $0.699791 \quad 2.247013-0.399617$ $\mathrm{N}-2.073842 \quad 2.127199-0.162950$ $\begin{array}{lll}\text { C -2.597851 } & 1.325218 & 0.834844\end{array}$ $\begin{array}{lll}C-1.839141 & 0.400173 & 1.448759\end{array}$ H $1.055799 \quad 1.3788320 .154293$ O $-0.263988 \quad 3.106187-1.171742$ $\mathrm{H}-2.6407622 .810322-0.636223$ $\mathrm{H}-3.642998 \quad 1.4821341 .068481$ $\mathrm{H}-2.238374-0.253608 \quad 2.209577$ N $\quad 0.433724-0.2669392 .032939$ $\begin{array}{llll}\mathrm{H} & 0.337954 & 0.419220 & 2.776329\end{array}$ H $1.391395-0.155278 \quad 1.670409$ O - $0.511967-0.875752-0.024807$

$\begin{array}{llll}O & 2.730979 & 0.541803 & 0.537935\end{array}$

C $3.551221-0.081125-0.213184$

C $4.536691 \quad 0.793637-0.988999$

O $3.630784-1.333313-0.383431$

H $3.983541 \quad 1.399843-1.706858$

H $\quad 5.269787 \quad 0.186361-1.514796$ H $\quad 5.036790 \quad 1.477914-0.304210$ Zn -2.420345 -1.525460 -0.859394

H $2.298589-2.1105010 .140058$ $\begin{array}{lll}\text { O } & 1.437117-2.543860 & 0.425538\end{array}$ H $1.383623-2.367369 \quad 1.373683$ H $\quad 0.274046-1.507956 \quad 0.068634$

Structure 17

C $0.729251-0.211109 \quad 0.489775$

N $1.233386-1.060445-0.626030$

C $2.316716-1.877499-0.656071$

N $3.115721-1.779170 \quad 0.492588$

C $3.014389-0.677515 \quad 1.350884$

$\begin{array}{llll}\text { C } & 1.923786 & 0.106044 & 1.357867\end{array}$

H $\quad 0.643257-1.074508-1.445842$

O $2.618195-2.647714-1.577436$

H $\quad 4.017589-2.2087450 .359536$

H $3.867742-0.514108 \quad 1.997066$

H $1.829626 \quad 0.934753 \quad 2.043495$

N -0.236181 $-0.953263 \quad 1.323242$

H $\quad 0.196918-1.826069 \quad 1.615920$ $\mathrm{H}-1.039496-1.189318 \quad 0.737440$

O $\quad 0.111703 \quad 0.879062-0.052374$

O $-3.005523-1.457305-0.055282$

C -3.928013 -0.655143 -0.203876

C $-5.162007-0.980988-1.016472$

O -3.988316 $0.568455 \quad 0.300880$

H $-5.223559-0.302090-1.865828$

H -6.053500 -0.830759 -0.409707

$\mathrm{H}-5.111812-2.007852-1.365596$

Zn $\quad 1.329902 \quad 2.325187-0.777225$

$\begin{array}{lll}\mathrm{H}-3.127916 & 0.806897 & 0.818551\end{array}$

$\begin{array}{llll}\mathrm{O}-1.901766 & 1.277433 & 1.584971\end{array}$

$\begin{array}{lll}\mathrm{H}-1.172932 & 1.316126 & 0.895768\end{array}$

$\mathrm{H}-1.552527 \quad 0.508042 \quad 2.068643$

Structure 18

C - $0.413613 \quad 0.060492 \quad 0.841166$

$\begin{array}{lll}\mathrm{N}-0.760917 & 1.370918 & 0.238499\end{array}$

$\mathrm{H}-0.016077 \quad 1.725334-0.351696$

$\begin{array}{llll}\mathrm{N} & 0.678766 & 0.396047 & 1.802815\end{array}$

$\begin{array}{llll}\mathrm{H} & 0.302534 & 0.948441 & 2.569318\end{array}$

H $\quad 1.780844 \quad 1.048028 \quad 0.906916$

O $0.080811-0.830255-0.053730$

$\begin{array}{llll}O & 2.360104 & 1.500508 & 0.160370\end{array}$

C $3.3306610 .688319-0.239066$

C $3.9902841 .180338-1.506653$

O $3.680434-0.346780 \quad 0.329266$

$\mathrm{Zn}-1.210011-1.618239-1.396976$

O $1.805512-2.280063 \quad 1.569475$

H $1.148140-2.0070350 .893771$

H $\quad 1.054974-0.487560 \quad 2.156325$

H $2.586246-1.796408 \quad 1.260149$

C - $1.639026-0.453253 \quad 1.554117$

C -2.000376 $1.763753-0.195044$

N -3.050209 $1.146107 \quad 0.478859$

$\begin{array}{lll}\text { C }-2.862911 & 0.038455 & 1.301421\end{array}$

O -2.205753 2.648059-1.033341

$\begin{array}{lll}\mathrm{H}-3.953575 & 1.327188 & 0.072697\end{array}$

H -3.767247 $-0.377042 \quad 1.727441$

$\mathrm{H}-1.512179-1.326826 \quad 2.178667$

H $\quad 4.894305 \quad 0.609666-1.697746$

H $3.293624 \quad 1.049652-2.334154$

H $4.2162932 .241846-1.425600$

Structure 19

$\begin{array}{lll}C-1.038206 & 0.538063 & 0.002603\end{array}$

$\begin{array}{lllll}\mathrm{N} & 0.236137 & 0.884778 & -0.279431\end{array}$

C $\quad 0.599369 \quad 2.175405-0.559544$

$\mathrm{N}-0.433014 \quad 3.134053-0.553773$
C -1.736510 $2.830539-0.281351$

C -2.083167 $1.550761-0.004177$

H $1.322235-0.240712-0.767932$

$\begin{array}{llll}\text { O } & 1.752762 & 2.553140 & -0.805777\end{array}$

H $-0.134300 \quad 4.079051-0.738122$

$\mathrm{H}-2.4396093 .653665-0.301692$

H -3.099338 $1.270045 \quad 0.222276$

$\begin{array}{llll}N & 2.817749 & 1.397882 & 2.137433\end{array}$

H $3.450180 \quad 0.645598 \quad 1.882201$

H $2.604854 \quad 1.849641 \quad 1.251764$

O - $1.323275-0.675472 \quad 0.292019$

H -0.076215 -0.980557 1.806966

O $0.627822-1.061486 \quad 2.467904$

H $1.401643-1.2524941 .917851$

H $\quad 1.9646620 .902956 \quad 2.387746$

O $1.932070-0.842473-1.332556$

C $2.800099-1.535113-0.615711$

C $3.742260-2.321858-1.500552$

$\begin{array}{lll}\text { O } & 2.873745-1.568221 & 0.613924\end{array}$

H $4.417172-2.910707-0.886575$

H $4.308869-1.635328-2.128295$

H $\quad 3.167087-2.971996-2.158141$

Zn -3.194714 -1.566562 -0.124979

\section{Structure TS7}

C - $-0.516171 \quad 0.126340 \quad 1.008455$

$\mathrm{N}-0.294848 \quad 1.439965 \quad 0.362760$

C - $1.213182 \quad 2.068327-0.434132$

$\mathrm{N}-2.529602 \quad 1.753525-0.102885$

C $-2.870988 \quad 0.840702 \quad 0.882916$

$\begin{array}{lll}C-1.935025 & 0.102516 & 1.503515\end{array}$

H $\quad 0.6646821 .563555 \quad 0.048967$

O $-0.955858 \quad 2.904114-1.307372$

H -3.227625 2.167282-0.697439

$\mathrm{H}-3.926585 \quad 0.774532 \quad 1.112867$

H -2.197472 -0.622698 2.259745

N $0.414018-0.008575 \quad 2.132190$

$\begin{array}{llll}\text { H } & 0.180730 & 0.728751 & 2.792498\end{array}$

$\begin{array}{llll}H & 1.335897 & 0.234648 & 1.759670\end{array}$

O $-0.308002-0.907142 \quad 0.098646$

\begin{tabular}{lllll}
\hline & 2.711212 & 0.918178 & 0.467868
\end{tabular}

C $3.5077550 .239658-0.216981$

C $4.549813 \quad 0.938271-1.074292$

O $3.549736-1.052950-0.296307$

H $4.285824 \quad 0.812754-2.124143$

H $\quad 5.524608 \quad 0.477722-0.922359$

H $4.587295 \quad 1.997540-0.835155$

Zn -1.922738 -1.623607 -0.990662

H $2.606004-1.595330 \quad 0.242963$

$\begin{array}{lll}\text { O } & 1.658878-2.167699 & 0.700258\end{array}$

H $1.621480-1.9855551 .649993$

H $\quad 0.719388-1.590290 \quad 0.366179$

\section{Structure TS8}

C $\quad 0.634714-0.1413350 .377315$

N $1.065457-1.077318-0.677995$

H $0.528555-1.025973-1.532973$

$\mathrm{N}-0.526666-0.754776 \quad 1.121061$

H - $-0.226358-1.555586 \quad 1.671175$

H - $1.270391-1.045706 \quad 0.452290$

O $0.212576 \quad 1.020910-0.167246$

O $-2.839845-1.228424-0.394973$

C $-3.803315-0.436600-0.224994$

C - $5.099866-0.712308-0.982495$

$\begin{array}{llll}O & -3.842845 & 0.610287 & 0.518056\end{array}$

Zn $1.668097 \quad 2.316169-0.747743$

$\begin{array}{llll}\mathrm{O}-1.753390 & 1.292434 & 1.647474\end{array}$ 
$\mathrm{H}-1.178060 \quad 1.635725 \quad 0.922614$

$\mathrm{H}-1.164526 \quad 0.253888 \quad 1.644194$

$\mathrm{H}-2.672972 \quad 1.006881 \quad 1.156942$

C $1.776239-0.015718 \quad 1.358188$

C $1.972457-2.088022-0.594029$

N $2.674623-2.102368 \quad 0.620785$

C $2.703654-0.982764 \quad 1.456705$

O $2.201582-2.925862-1.472191$

H $\quad 3.480830-2.705967 \quad 0.584518$

H $3.516224-0.956088 \quad 2.171596$

H $\quad \begin{array}{lll}1.777470 & 0.840349 & 2.016009\end{array}$

H -5.939336 -0.708392 -0.288206

H -5.269671 $0.087963-1.702942$

H -5.046516 -1.665213 -1.502983

\section{Structure TS9}

$\begin{array}{llll}C & 0.664181 & 0.101011 & 0.473885\end{array}$

$\mathrm{N} \quad 0.991973-0.766999-0.601277$

C $2.154301-1.490108-0.720721$

N $3.171600-1.073735 \quad 0.144314$

C $3.042158-0.013325 \quad 1.022666$

C $\quad 1.847833 \quad 0.579235 \quad 1.213544$

H $\quad 0.150940-1.212371-1.006379$

O $2.349746-2.395964-1.531039$

H $4.066645-1.496916-0.037283$

H $3.947998 \quad 0.293147 \quad 1.529508$

H $1.7159361 .393760 \quad 1.908438$

$\mathrm{N}-0.066686-1.233574 \quad 1.726237$

H $0.591429-1.8228152 .225653$

H -0.643750 -1.782076 1.056117

$\begin{array}{lll}0-0.335281 & 0.882374 & 0.297586\end{array}$

O -1.394235 -2.065368 -0.532892

C $-2.512223-1.433678-0.495202$

C -2.970853 -0.826533-1.820801

O $-3.229351-1.244050 \quad 0.522599$

$\mathrm{H}-2.434399 \quad 0.114254-1.957384$

H -4.039317 -0.620871-1.804842

$\mathrm{H}-2.719504-1.482603-2.652895$

Zn $\quad 0.040044 \quad 2.704240-0.730716$

$\mathrm{H}-2.725441-0.134967 \quad 1.897880$

$\begin{array}{lll}\mathrm{O}-2.233562 & 0.502659 & 2.456096\end{array}$

$\begin{array}{lll}\mathrm{H}-1.698495 & 0.948285 & 1.779758\end{array}$

$\mathrm{H}-0.702496-0.740674 \quad 2.358961$ 